Aus der Abteilung Kardiologie und Pneumologie

(Prof. Dr. med. G. Hasenfuß)

im Zentrum Innere Medizin

der Medizinischen Fakultät der Universität Göttingen

\title{
Über die Prävalenz der Cheyne-Stokes-Atmung bei herzinsuffizienten Patienten unter moderner medikamentöser Therapie
}

\author{
INAUGURAL-DISSERTATION \\ zur Erlangung des Doktorgrades \\ der Medizinischen Fakultät \\ der Georg-August-Universität zu Göttingen
}

vorgelegt von

Dennis Beil

aus

Berlin

Göttingen 2011 
Dekan: Prof. Dr. med. C. Frömmel

1. Berichterstatter: Prof. Dr. med. S. Andreas

2. Berichterstatter: Priv.-Doz. Dr. med. A. Bräuer

3. Berichterstatterin: Prof. Dr. rer. nat. P. Virsik-Köpp

Tag der mündlichen Prüfung: 6. Februar 2012 


\section{Inhaltsverzeichnis}

1. Einleitung

Seite 5

1.1. Die Cheyne-Stokes-Atmung Seite 5

1.1.1. Geschichte der Cheyne-Stokes-Atmung Seite 5

1.1.2. Definition der Cheyne-Stokes-Atmung Seite 6

1.1.3. Ätiologie der Cheyne-Stokes-Atmung Seite 7

1.1.4. Normale Schlafarchitektur und Schlafstörungen durch Cheyne-Stokes-Atmung Seite 9

1.1.5. Therapie der Cheyne-Stokes-Atmung Seite 12

1.2. Die Herzinsuffizienz Seite 16

1.2.1. Definition, Ätiologie und Epidemiologie Seite 16

1.2.2. Klinik und Diagnostik Seite 17

1.3. Cheyne-Stokes-Atmung und Herzinsuffizienz Seite 18

1.4. Medikamentöse Therapie der chronischen Herzinsuffizienz Seite 19

1.4.1. Allgemeines Seite 19

1.4.2. ACE-Hemmer und AT1-Antagonisten Seite 21

1.4.3. B-Adrenozeptorantagonisten Seite 22

1.4.4. Diuretika Seite 22

1.4.5. Aldosteronantagonisten Seite 23

1.4.6. Digitalisglykoside Seite 23

1.5. Fragestellung Seite 24

$\begin{array}{ll}\text { 2. Methodik } & \text { Seite } 25\end{array}$

2.1. Patientenkollektiv Seite 25

2.1.1. Einschlusskriterien Seite 25

2.1.2. Ausschlusskriterien Seite 25

2.2. Ablauf der Untersuchung Seite 26

2.3. Polysomnografische Untersuchung Seite 27 
2.4. Auswertung Seite 29

2.4.1. Schlafstadien Seite 29

2.4.2. Atmung Seite 29

2.4.3. Statistik Seite 30

3. Ergebnisse $\quad$ Seite 31

3.1. Gesamtes Patientenkollektiv Seite 31

3.1.1. Allgemeine Patientendaten, Echokardiografie, EKG $\begin{array}{ll}\text { und Lungenfunktion } & \text { Seite } 31\end{array}$

3.1.2. Polysomnografische Untersuchung Seite 32

3.1.3. Prävalenz der Cheyne-Stokes-Atmung Seite 32

3.2. Patientengruppen mit und ohne Cheyne-Stokes-Atmung Seite 33

3.2.1. Patientengruppen Seite 33

3.2.2. Echokardiografie, EKG und Lungenfunktion Seite 35

$\begin{array}{ll}\text { 3.2.3. Polysomnografische Daten } & \text { Seite } 37\end{array}$

3.3. Vergleich der polysomnografischen Daten der Patienten mit einer EF größer und kleiner als 25 Prozent Seite 39

4. Diskussion

Seite 41

4.1. Prävalenz der Cheyne-Stokes-Atmung Seite 41

4.2. Einfluss der medikamentösen Herzinsuffizienztherapie Seite 47

4.3. Weitere Erkenntnisse Seite 49

5. Zusammenfassung Seite 51

6. Anhang Seite 52

6.1. Tabellen Seite 52

6.2. Patientenaufklärung zur Studie Seite 54

6.3. Einverständniserklärung des Patienten Seite 55

6.4. Abkürzungen Seite 56

$\begin{array}{ll}\text { 7. Literaturverzeichnis } & \text { Seite } 57\end{array}$ 


\section{Einleitung}

\subsection{Die Cheyne-Stokes-Atmung}

\subsubsection{Geschichte der Cheyne-Stokes-Atmung}

Die Cheyne-Stokes-Atmung wurde erstmalig im Jahre 1818 von dem Arzt John Cheyne beschrieben. Er berichtete von einem Patienten mit Herzschwäche, dessen Atmung für etwa eine Viertelminute sistierte, dann graduell bis zu einem Maximum an Atemtiefe anstieg, um dann ebenso graduell wieder abzunehmen bis zum erneuten Atemstillstand. Dieser Atemtyp habe über acht bis neun Tage angehalten (Cheyne 1818). Der Arzt William Stokes beschrieb 1854 das gleiche Phänomen und brachte es in Zusammenhang mit Patienten mit "fatty degeneration of the heart". Die Patienten mit diesem Atemphänomen verstarben alsbald (Stokes 1854). Deshalb galt die Cheyne-Stokes-Atmung als prognostisch infaustes Zeichen.

Im Jahr 1961 wurde von Brown und Plum bestätigt, dass die Cheyne-StokesAtmung in hoher Koinzidenz mit der Herzinsuffizienz auftritt (Brown und Plum 1961). Zusätzlich wurde dieser Atemtyp jedoch auch bei einer Vielzahl neurologischer Erkrankungen (Garlind und Linderholm 1958, Karp et al. 1961) sowie bei Früh- und Neugeborenen beobachtet (Howard und Bauer 1949, Chernick und Avery 1966). Während bei letzteren Patienten die Cheyne-StokesAtmung mit der Reifung des kindlichen Gehirns verschwindet (Cross et al. 1953), ergaben sich Hinweise darauf, dass sie bei zugrunde liegender neurologischer oder kardiologischer Erkrankung mit einer schlechten Prognose des Patienten behaftet ist (Dowell et al. 1971).

Mehrere Untersuchungen konnten zeigen, dass bei herzinsuffizienten Patienten die Prävalenz der Cheyne-Stokes-Atmung in der Nacht höher ist als am Tage oder eine bestehende Cheyne-Stokes-Atmung im Schlaf noch deutlicher hervortritt (Harrison et al. 1934, Dowell et al. 1971). Zur genaueren Beschreibung der nächtlichen Atemstörungen verwendeten Dark et al. 1987 erstmals die Technik der Polysomnografie (Dark et al. 1987). Hiernach ergaben weiterführende Studien, dass der nächtliche Ausprägungstyp der Cheyne- 
Stokes-Atmung entgegen der am Tage prävalenten Form nur selten mit einer unmittelbar infausten Prognose verbunden zu sein scheint (Andreas et al. 1996 b), Patienten mit Herzinsuffizienz und nächtlicher Cheyne-Stokes-Atmung jedoch eine schlechtere Überlebensrate als eine vergleichbare Patientengruppe ohne diese Atemstörung haben (Ancoli-Israel et al. 1994, Hanly und ZuberiKhokar 1996, Lafranchi et al. 1999).

\subsubsection{Definition der Cheyne-Stokes-Atmung}

Es herrscht in der Literatur Uneinigkeit darüber, wie die Cheyne-Stokes-Atmung zu definieren sei. Die Erstbeschreiber John Cheyne und William Stokes beschrieben sie als periodische Crescendo-Decrescendo-Atmung, zwischen deren Zyklen es zu einem völligen Sistieren der Atmung komme (Cheyne 1818, Stokes 1854). Später wurde der Begriff vor allem in der britischen Literatur für alle periodischen Atmungsformen mit Crescendo-Decrescendo-Charakter verwandt, auch wenn diese keine Apnoephasen enthielten (Younes 1989). In der vorliegenden Arbeit wurde die Cheyne-Stokes-Atmung nach den Kriterien der American Academy of Sleep Medicine von 2005 definiert. Hiernach gelten folgende diagnostische Parameter:

A. Eigen- oder fremdanamnestisch leidet der Patient unter Schlaflosigkeit oder exzessiver Tagesmüdigkeit.

B. Während des Schlafes treten häufig Hypopnoen oder Apnoen auf.

C. Im Schlaf tritt mindestens einer der folgenden Faktoren auf:

1. Das Gefühl der Luftnot oder des Erstickens

2. Häufige Körperbewegungen

3. Eine Zyanose. 
D. In der Polysomnografie zeigen sich

1. überwiegend zentrale Apnoen von mindestens zehn Sekunden Dauer in Verbindung mit

a. häufigen Aufwachreaktionen (sog. Arousels) in Zusammenhang mit Apnoephasen

und/oder

b. Abfällen der Sauerstoffsättigung in Zusammenhang mit Apnoephasen

und/oder

c. Bradytachykardien

2. fakultativ ein multipler Schlaf-Latenz-Test (MSLT) mit einer durchschnittlichen Schlaflatenz von weniger als zehn Minuten.

E. Vergesellschaftung mit anderen Schlafstörungen.

Erforderlich für die Diagnose einer Cheyne-Stokes-Atmung sind die Kriterien A, B und D (American Academy of Sleep Medicine 2005).

\subsection{3. Ätiologie der Cheyne-Stokes-Atmung}

Die Entstehung der Cheyne-Stokes-Atmung lässt sich auf viele, zum Teil synergistisch wirkende Mechanismen zurückführen. Diese Mechanismen lassen sich zu einem zusammenhängenden System zusammenfügen: dem Regelkreis der Atmung (Cherniak und Longobardo 1973). Dieser besteht aus dem arteriellen Kohlendioxidpartialdruck und der arteriellen Sauerstoffsättigung als geregelte Größen, den Chemozeptoren der Aorta und der Karotisgabel als Messfühler und dem Atemzentrum als Regelorgan. Veränderungen der Blutgaskonzentration erreichen über die Blutzirkulation die Messfühler. Nach Meldung dieser Veränderungen an das Atemzentrum kann dieses durch eine adäquate und schnelle Reaktion die Atmung entsprechend regulieren und den Regelkreis stabil halten. Die Funktionsweise des Regelkreises konnte bereits in 
mathematischen Modellen beschrieben werden (Khoo et al. 1991). Die CheyneStokes-Atmung entsteht durch Störungen dieses Regelkreises, die auf verschiedene Ursachen zurückgeführt werden können. Die wichtigsten werden im Weiteren kurz erläutert.

1. Die Atemkontrolle im Schlaf erfolgt vor allem metabolisch über den arteriellen Partialdruck des Sauerstoffs $\left(\mathrm{pO}_{2}\right)$ und des Kohlendioxids $\left(\mathrm{pCO}_{2}\right)$, wobei eine Erniedrigung des $\mathrm{pCO}_{2}$ den Atemantrieb inhibiert, während eine Erniedrigung des $\mathrm{pO}_{2}$ den Atemantrieb stimuliert (Berger et al. 1977). Beim Übergang vom Wachzustand in den Schlaf findet durch alveoläre Hypoventilation eine Erhöhung des $\mathrm{pCO}_{2}$ statt. Parallel dazu steigt die Apnoe-Schwelle an, also der $\mathrm{pCO}_{2}$-Grenzwert, unter dem vom Atemzentrum zur Steigerung des $\mathrm{pCO}_{2}$ eine Apnoe induziert wird. Auf eine Erniedrigung des $\mathrm{pCO}_{2}$ reagiert das Atemzentrum dann mit relativ starker Inhibition der Atmung. Menschen mit niedrigem $\mathrm{pCO}_{2} \mathrm{im}$ Wachen liegen vor allem beim Übergang in den Schlaf unter der Apnoeschwelle (Dempsey und Skatrud 1986). Es konnte gezeigt werden, dass Patienten mit Cheyne-Stokes-Atmung sowohl im Wachzustand als auch im Schlaf niedrigere $\mathrm{pCO}_{2}$-Werte haben als Patienten ohne diese Atemstörung (Brown und Plum 1961, Lange und Hecht 1961, Dowell et al. 1971, Naughton et al. 1993). Weil Patienten mit im Wachzustand gegenüber der Norm erniedrigtem $\mathrm{pCO}_{2}$ im Schlaf häufig Apnoen entwickeln, gilt der $\mathrm{pCO}_{2}$ im Wachzustand als bester Prädiktionsfaktor für die Entwicklung einer Cheyne-Stokes-Atmung im Schlaf (Hanly et al. 1993, Javaheri und Corbett 1998, Sin et al. 1999).

2. Eine Verlängerung der Blutzirkulationszeit von der Lunge zu den Messfühlern der Aorta und $A$. carotis führt zu einer verspäteten Registrierung der veränderten arteriellen Atemgase mit konsekutiv verzögerter Korrektur der Atemregulation durch das Atemzentrum. Dies begünstigt Oszillationen der Atmung (Klein 1930, Pryor 1951). Es konnte gezeigt werden, dass eine künstliche Verlängerung der Zirkulationszeit zu einer Cheyne-Stokes-Atmung führt (Crowell et al. 1956). 
3. Veränderungen der Sauerstoff- und Kohlendioxidpartialdrücke werden auch durch die Größe der Gasspeicher des Körpers mitbestimmt. Für eine Änderung des $\mathrm{pCO}_{2}$ sind aufgrund der großen $\mathrm{CO}_{2}$-Anteile in Lunge, Blut und Gehirn größere Atmungsveränderungen nötig als für Änderungen des $\mathrm{pO}_{2}$, da die Dämpfungseigenschaften der Sauerstoffspeicher geringer sind. Eine Hypoxie durch geringe Atemschwankungen führt über die oben genannten Mechanismen zu einer Hypokapnie mit konsekutiver Apnoe. Es wurde gezeigt, dass Zustände einer kombinierten Hypoxie mit Hypokapnie eine oszillierende Atmung begünstigen (Cherniak und Longobardo 1970, Cherniak und Longobardo 1973).

\subsubsection{Normale Schlafarchitektur und Schlafstörungen durch Cheyne- Stokes-Atmung}

Nach den Kriterien von Rechtschaffen und Kales (Rechtschaffen und Kales 1968) werden anhand der Ableitungen des Elektroenzephalogramms (EEG), des Elektrookulogramms (EOG) und des Mundboden-Elektromyogramms (EMG) sechs verschiedene Schlafstadien unterschieden.

Das Stadium Wach entspricht dem Wachzustand. In inm herrscht ein niedergespanntes, gemischtfrequentes EEG mit hauptsächlich $\alpha$-Aktivität (8-13 Hz) vor. Im EOG finden sich schnelle Augenbewegungen (Rapid Eye Movements (REM)), das EMG ist relativ hochgespannt.

Stadium 1 ist definiert durch ein niedergespanntes (50-75 $\mu \mathrm{V})$ EEG mit einer Wellenfrequenz von 2-7 Hz. Gelegentlich treten in diesem Stadium vereinzelt steilzackige Wellen, sogenannte Vertexwellen, mit einer Amplitude von bis zu $200 \mu \mathrm{V}$ auf. Ebenfalls charakteristisch für das Schlafstadium 1 sind langsame, bis zu mehreren Sekunden dauernde Augenbewegungen. Das EMG-Niveau liegt unter dem des entspannten Wachseins.

In Stadium 2 zeigt sich ein ähnliches EEG wie in Stadium 1, jedoch treten hier zusätzlich K-Komplexe (Wellen mit einer gut abgrenzbaren, negativen scharfen Welle, auf die unmittelbar eine positive Komponente folgt) und Schlafspindeln (Perioden $>0,5$ Sekunden, die durch Wellen mit einer Frequenz von $12-14 \mathrm{~Hz}$ gekennzeichnet sind) auf. Ebenfalls kommen in diesem Stadium im EEG Wellen 
höherer Amplitude und niedrigerer Frequenz ( $\delta$-Wellen) vor, jedoch nicht genug, um Stadium 3 oder 4 zu definieren.

Stadium 3 ist definiert durch ein EEG, das in einer 30-Sekunden-Epoche mindestens 20 Prozent, jedoch nicht mehr als 50 Prozent der Aufzeichnung $\delta$-Wellen mit einer Frequenz von $0-4 \mathrm{~Hz}$ und einer Amplitude von mindestens 75 $\mu \mathrm{V}$ aufweist.

In Stadium 4 beträgt der Anteil der $\delta$-Wellen am EEG einer 30-SekundenEpoche mindestens 50 Prozent.

Die Stadien 1 bis 4 werden unter dem Begriff Non-REM-Schlaf (NREM) zusammengefasst und damit dem Stadium REM gegenübergestellt.

Das Stadium REM ist definiert durch ein niedergespanntes, gemischtfrequentes EEG und das Auftreten von schnellen Augenbewegungen. Zusätzlich erreicht die EMG-Ableitung in diesem Stadium ihr niedrigstes Niveau verglichen mit allen anderen Schlafphasen.

Beim Gesunden vertieft sich der Schlaf stufenweise aus den Leichtschlafstadien 1 und 2 in die Tiefschlafstadien 3 und 4 . Schließlich wird das Stadium REM durchlaufen, mit dem ein kompletter Schlafzyklus endet. Ein gesunder Erwachsener durchläuft in acht Stunden Schlaf etwa vier dieser Schlafzyklen mit einem Anteil des Stadiums REM von 20 bis 23 Prozent an der Gesamtschlafzeit (Birbaumer und Schmidt 1997).

Mit der Cheyne-Stokes-Atmung ist eine Störung der normalen Schlafarchitekur verbunden (Ancoli-Israel et al. 1994). Diese Störung ist durch Abnahme der Tiefschlafstadien und Zunahme der Leichtschlafstadien sowie eine Rarifizierung der REM-Stadien charakterisiert. Weiterhin kommt es bei der nächtlichen Cheyne-Stokes-Atmung zu häufigen Abfällen der kapillären Sauerstoffsättigung sowie zu vermehrten Aufwachreaktionen (Arousals), die vorwiegend in der hyperventilatorischen Phase des periodischen Atemzyklus vorkommen und zu einer Fragmentierung des Schlafs führen. Diese Fragmentierung hat eine verminderte Schlafqualität, eine erhöhte Tagesmüdigkeit und eine Abnahme der Leistungsfähigkeit zur Folge (Hanly und Zuberi-Khokar 1995). Die Abbildung 1 zeigt das Hypnogramm eines Patienten ohne signifikante nächtliche Atemstörungen, Abbildung 2 stellt die typische Schlafstadienverteilung eines Patienten mit Cheyne-Stokes-Atmung dar. 
Abbildung 1: Hypnogramm eines Patienten ohne signifikante nächtliche Atemstörungen.

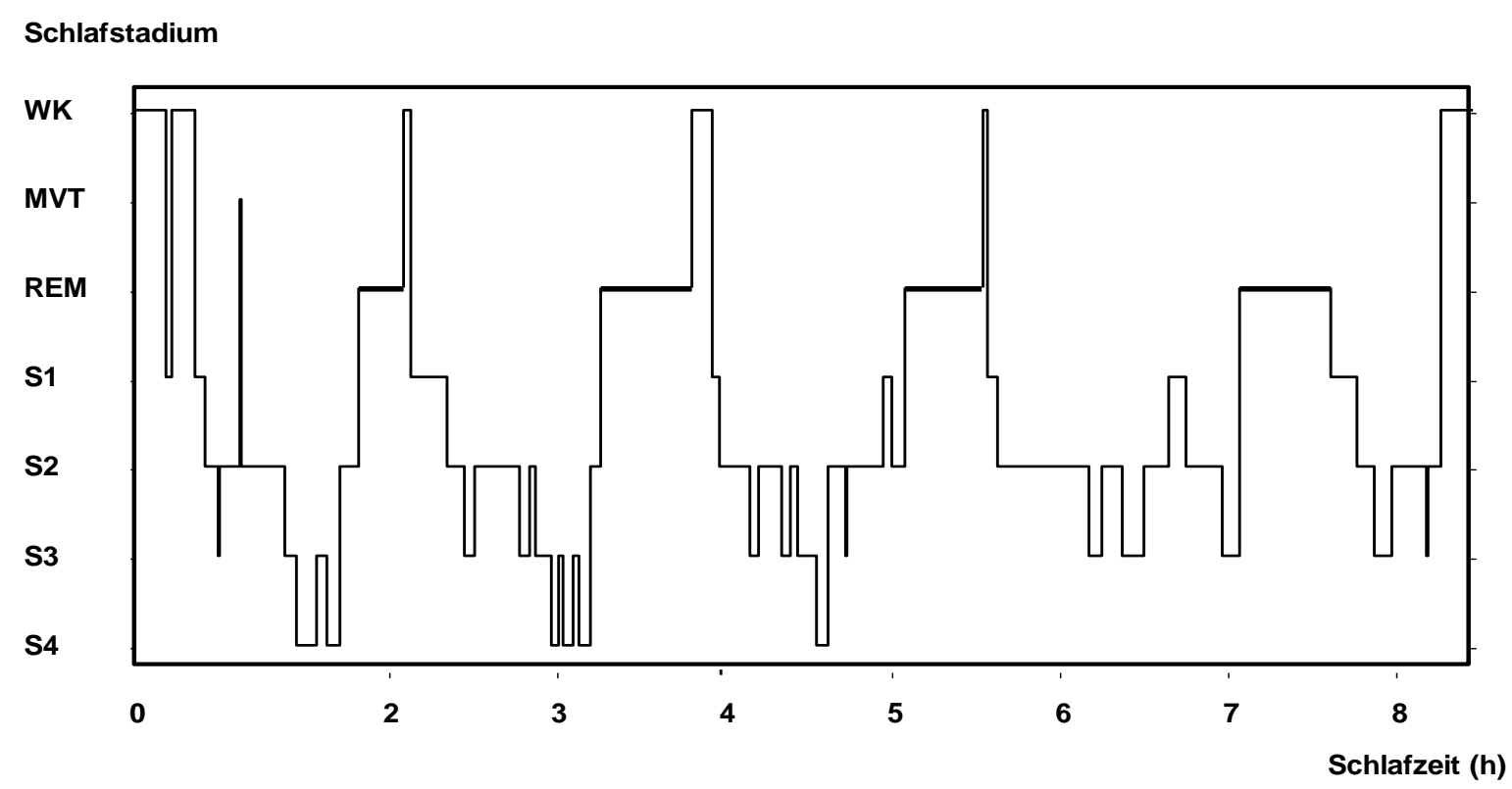

WK= Wachstadium, MVT= Movement Time, REM= Stadium Rapid Eye Movement, S1-4= Schlafstadium 1-4.

Abbildung 2: Hypnogramm eines Patienten mit Cheyne-Stokes-Atmung.

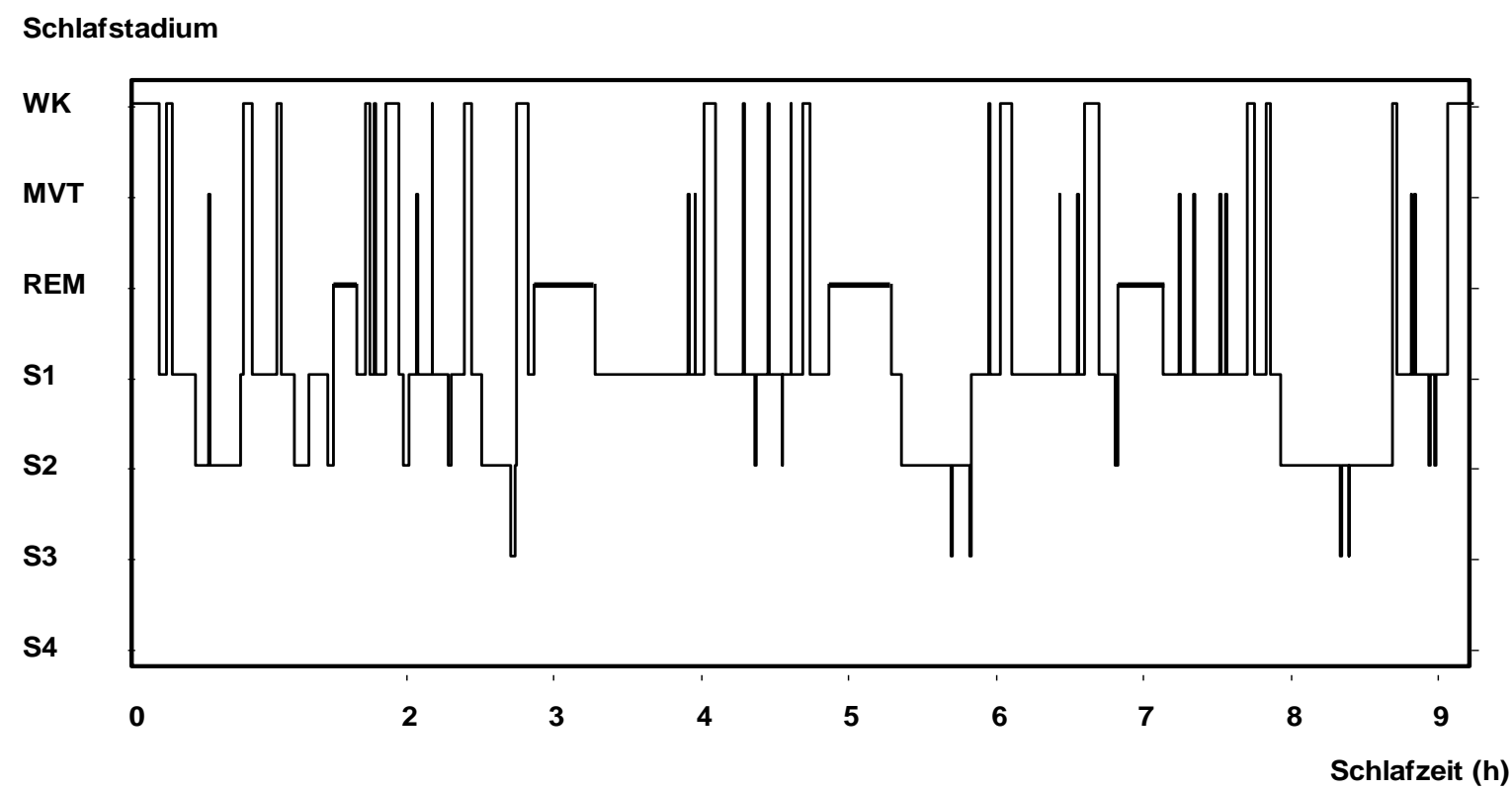

WK= Wachstadium, MVT= Movement Time, REM= Stadium Rapid Eye Movement, S1-4= Schlafstadium 1-4. 


\subsubsection{Therapie der Cheyne-Stokes-Atmung}

Es existieren verschiedene Ansätze zur Therapie der Cheyne-Stokes-Atmung. Harrison et al. konnten bereits 1934 zeigen, dass durch Therapie eines kardialen Lungenödems ein Rückgang der Cheyne-Stokes-Atmung zu erwarten ist (Harrison et al. 1934).

In zahlreichen Studien konnte eruiert werden, dass die nächtliche Applikation von Sauerstoff zu einem signifikanten Rückgang der nächtlichen Atemstörungen sowie einer Anhebung der durchschnittlichen kapillären Sauerstoffsättigung führt (Hanly et al. 1989, Walsh et al. 1995, Javaheri et al. 1999, Krachmann et al 1999). Auch zeigte sich, daß die nächtliche Sauerstoffinsufflation die Atemantwort auf erhöhte Koheldioxid-Partialdrücke dämpft und damit der Entstehung einer Cheyne-Stokes-Atmung entgegenwirken kann (Andreas et al. 1998 a). Die nächtliche Sauerstoffgabe verbessert zudem die kognitive und körperliche Leistungsfähigkeit von Patienten mit Cheyne-Stokes-Atmung am Tage (Andreas et al. 1996 a). Hinsichtlich der Schlafqualität ergaben verschiedene Studien keine einheitlichen Ergebnisse. Denn während einige Studien diesbezüglich keine Vorteile einer nächtlichen Sauerstofftherapie herausarbeiten konnten (Staniforth et al. 1998, Franklin et al. 1997), kam es in anderen Untersuchungen parallel zu rückläufigen Atemstörungen auch zu einer signifikanten Verbesserung des Schlafprofils mit Zunahme der Tiefschlafstadien (Hanly et al. 1989, Walsh et al. 1995, Andreas et al. 1998 b, Javaheri et al. 1999). Eine signifikante Verbesserung der kardialen Pumpfunktion wurde unter einer Sauerstofftherapie trotz gemilderter Atemstörungen jedoch nicht beobachtet (Arzt et al. 2005). Eine Studie berichtet sogar von einer erhöhten kardialen Nachlast bei Hyperoxie (Mak et al. 2001). Trotzdem wird eine nächtliche Sauerstofftherapie unter polygraphischer Kontrolle derzeit als eine mögliche Option zur Therapie einer nächtlichen Cheyne-Stokes-Atmung angesehen.

Nach nächtlicher Applikation von $\mathrm{CO}_{2}$ konnte durch Anhebung des $\mathrm{pCO}_{2}$ über die Apnoeschwelle eine Verminderung nächtlicher Atemstörungen erreicht 
werden (Lorenzi-Filho et al. 1999). Da jedoch eine Erhöhung der Sympathikusaktivität mit den entsprechenden Auswirkungen auf das HerzKreislauf-System unter dieser Therapie beschrieben ist (Andreas et al. 1998 b), wird diese Form der Beatmung speziell für Patienten mit Herzinsuffizienz nicht empfohlen.

In einer Studie führte die Verabreichung von Theophyllin zu einer Milderung nächtlicher zentraler Atemstörungen und Erhöhung der durchschnittlichen Sauerstoffsättigung im Schlaf (Javaheri et al. 1996). Eine Verbesserung der Schlafarchitektur oder der linksventrikulären Ejektionsfraktion wird hierdurch jedoch nicht herbeigeführt (Dowdell et al. 1990). Zudem konnten Andreas et al. unter Therapie mit Theophyllin neben einer Erhöhung des Atemminutenvolumens auch eine Erhöhung der Reninaktivität im Plasma feststellen (Andreas et al. 2004) mit möglichen nachteiligen Effekten für das kardiovaskuläre System herzinsuffizienter Patienten. Suissa et al. fanden eine erhöhte Sterblichkeit in einem Kollektiv herzinsuffizienter Patienten, die wegen einer Lungenerkrankung mit Theophyllin behandelt wurden (Suissa et al. 1996). Von der Mehrzahl der Autoren wird deshalb die Langzeitgabe von Theophyllin bei Patienten mit Herzinsuffizienz abgelehnt.

Es konnte ein Rückgang nächtlicher Atemstörungen sowie eine Verbesserung der linksventrikulären Ejektionsfraktion durch eine nasale CPAP-Therapie (Continuous Positive Airway Pressure) herbeigeführt werden (Naughton et al. 1995). Ebenso wurde eine Verminderung der Mortalität an kardiovaskulären Ereignissen und der Notwendigkeit einer Herztransplantation bei Patienten mit Herzinsuffizienz und nächtlicher Cheyne-Stokes-Atmung durch diese Beatmungstherapie festgestellt (Sin et al. 2000). Andere Studien konnten diese Ergebnisse unter ähnlichen Bedingungen jedoch nicht oder nur teilweise zeigen (Buckle et al. 1992, Arzt et al. 2006). Bradley stellte 1992 zudem eine Verminderung des Herzzeitvolumens unter CPAP-Therapie fest und sah hierdurch die Entstehung einer Cheyne-Stokes-Atmung sogar begünstigt (Bradley 1992). Eine Studie berichtet auch von einem Todesfall unter dieser Therapie (Davies et al. 1993). In der bisher größten randomisierten und 
kontrollierten Studie zur CPAP-Therapie bei Herzinsuffizienz und einhergehenden zentralen Atemstörungen, der CANPAP-Studie, konnten die nächtlichen zentralen Atemstörungen durch eine CPAP-Therapie signifikant gesenkt werden. Weiterhin zeigten sich eine Besserung der linksventrikulären Ejektionsfraktion und der nächtlichen Sauerstoffsättigung sowie eine Verminderung des Norepinephrinspiegels im Plasma als indirektem Marker für die Symphatikusaktivität. Jedoch kam es unter der Therapie zu keiner Verminderung der Mortalität, vielmehr gab es in den ersten Monaten nach Therapiebeginn sogar einen Trend hin zu einer erhöhten Sterblichkeit verglichen mit der Kontrollgruppe ohne CPAP-Therapie (Bradley et al. 2005). Auch wenn eine Subgruppenanalyse der CANPAP-Studie bei einer Reduktion des Apnoe-Hypopnoe-Index (AHI) unter $15 / \mathrm{h}$ eine Besserung der Überlebensrate zeigen konnte (Arzt et al. 2007), hat die nasale CPAP-Therapie derzeit keinen Stellenwert in der Behandlung zentraler Atemstörungen und ist im wesentlichen Patienten mit obstruktivem Schlafapnoesyndrom vorbehalten. Die Beatmung im Bi-Level-Modus, einer Weiterentwicklung der konventionellen CPAP-Beatmung, zeigte im Vergleich zur CPAP-Therapie keine Überlegenheit bezüglich der polysomnografischen und klinischen Parameter (Köhnlein et al. 2002), so dass dieser Beatmungmodus nur für Patienten empfohlen wird, die eine Beatmung im CPAP-Modus nur unzureichend tolerieren (Pevernagie et al. 2007).

Eine Vielzahl neuerer Studien untersuchte den Effekt der adaptiven Servoventilation (ASV) auf die Cheyne-Stokes-Atmung. Bei dieser Form der nichtinvasiven Beatmung erfolgt eine bedarfsadaptierte Modifikation der Beatmungsdrücke im Falle eines Abfalls der patienteneigenen Atmung. Mehrere Studien konnten zeigen, dass ASV eine periodische Atmung effektiv vermindern kann (Teschler et al. 2001, Töpfer et al. 2004, Philippe et al. 2006, Morgenthaler et al. 2007). Teschler et al. stellten in einer vergleichenden Studie fest, dass durch ASV neben den Atemstörungen auch das Schlafprofil gebessert wird und diese Therapieform bezüglich beider Parameter der Sauerstoffgabe, der CPAPund der Bilevel-Therapie überlegen ist (Teschler et al. 2001). In mehreren Untersuchungen konnte vor allem nach längerfristiger Therapie eine Besserung der Lebensqualität und der körperlichen Leistungsfähigkeit belegt werden (Töpfer et al. 2004, Philippe et al. 2006). Auch wurde bei Pepperell et al. in einer 
randomisierten Studie ein deutlicher Rückgang der Tagesmüdigkeit nach effektiver ASV-Therapie festgestellt (Pepperell et al. 2003). Die ASV-Therapie wies in allen vergleichenden Studien die beste Patientencompliance auf (Teschler et al. 2001, Philippe et al. 2006, Morgenthaler et al. 2007). Im Gegensatz zu den unterschiedlichen Ergebnissen der Untersuchungen zur CPAP-Therapie konnte unter langfristiger Anwendung der ASV bisher einheitlich eine signifikante Verbesserung der kardialen Leistungsfähigkeit und der linksventrikulären Ejektionsfraktion nachgewiesen werden (Schädlich et al. 2004, Philippe et al. 2006). Auch zeigte sich ein signifikanter Rückgang des Serum-BNP (Brain Natriuretic Peptide) und der Metadrenalinausscheidung im Urin (Pepperell et al. 2003), so dass sich auch laborchemisch Hinweise für eine Besserung der Herzinsuffizienz sowie eine Verminderung der Sympathikusaktivität unter ASV-Therapie ergeben. Es gibt bisher jedoch noch keine Studie, die einen positiven Effekt dieser Therapie auf die Mortalität von Patienten mit Herzinsuffizienz und Cheyne-Stokes-Atmung aufzeigt.

Garrigue et al. zeigten 2002, dass eine Erhöhung der Herzfrequenz durch atriale Schrittmacherstimulation zu einem signifikanten Rückgang nächtlicher zentraler Atemstörungen führt (Garrigue et al. 2002). Spätere Studien ergaben zudem eine Besserung der Cheyne-Stokes-Atmung nach Implantation eines biventrikulären Schrittmachersystems, wobei der Rückgang nächtlicher zentraler Atemstörungen hier vor allem auf die Besserung der kardialen Pumpfunktion zurückgeführt wurde (Sinha et al. 2004, Oldenburg et al. 2007, Lüthje et al. 2009). Die Kombination eines biventrikulären Schrittmachersytems mit einer atrialen Überstimulation erbrachte einen weiteren Rückgang nächtlicher zentraler Atemstörungen (Lüthje et al. 2009).

$\mathrm{Zu}$ keiner der genannten Behandlungsmethoden existieren derzeit Langzeitergebnisse bezüglich der Auswirkungen auf die Mortalitätsrate von Patienten mit Herzinsuffizienz, so dass es zur Therapie nächtlicher zentraler Atemstörungen derzeit keine einheitlichen Behandlungsrichtlinien gibt. 


\subsection{Die Herzinsuffizienz}

\subsubsection{Definition, Ätiologie und Epidemiologie}

Definitionsgemäß handelt es sich bei der Herzinsuffizienz um einen pathophysiologischen Zustand, in dem die Herzfunktion nicht ausreicht, um die peripheren Gewebe in Ruhe und bei Belastung mit ausreichend Sauerstoff zu versorgen (Deutsche Gesellschaft für Kardiologie 2005).

Die Ätiologie der Herzinsuffizienz kann sehr unterschiedlich sein. Die häufigsten Ursachen in westlichen Ländern sind die koronare Herzkrankheit und die arterielle Hypertonie (McKee et al. 1971). Andere häufige Ursachen sind der stattgehabte Myokardinfarkt, Herzrhythmusstörungen und Herzklappenfehler (European Society of Cardiology Task Force 2001).

Die Prävalenz der Herzinsuffizienz beträgt 3-20/1000 Menschen und steigt mit dem Alter auf über 100/1000 bei über 65-jährigen. Dabei ist die Prävalenz bei Männern 1,5 mal so hoch wie bei Frauen (McMurray und Stewart 2000). Die Prognose bei Herzinsuffizienz ist schlecht. Die Mortalität beträgt im ersten Jahr nach Diagnosestellung 24 Prozent (Gustafsson et al. 2003), die Fünf-JahresÜberlebensrate 40-60 Prozent (Levy et al. 2002). In den NYHA-Stadien III und IV liegt die Ein-Jahres-Überlebensrate bei nur 62 Prozent (Cowie et al. 2000). 


\subsubsection{Klinik und Diagnostik}

Die Hauptsymptome der Herzinsuffizienz sind die Verminderung der allgemeinen körperlichen Leistungsfähigkeit, Kurzatmigkeit und Müdigkeit. Weitere führende klinische Symptome sind Unterschenkelödeme, Tachykardie und nächtlicher Husten (Rihal et al. 1995). Durch die New York Heart Association wurde die Herzinsuffizienz nach diesen Kriterien in vier Schweregrade eingeteilt (NYHA 1964):

NYHA-Klasse I Beschwerdefreiheit, normale körperliche Belastungsfähigkeit

NYHA-Klasse II Beschwerden bei starker körperlicher Belastung

NYHA-Klasse III Beschwerden bei leichter körperlicher Belastung

NYHA-Klasse IV Beschwerden in Ruhe.

Neben der klinischen Diagnostik anhand der Symptome spielen verschiedene Routineverfahren wegen ihrer allgemeinen Verfügbarkeit und geringen Invasivität eine wichtige Rolle in der Diagnostik und Verlaufsbeurteilung der Herzinsuffizienz.

Als wichtigste apparative Methode zur Beurteilung der Herzfunktion gilt die Echokardiografie (European Society of Cardiology Task Force 2001, Deutsche Gesellschaft für Kardiologie 2005). Mit ihrer Hilfe kann die linksventrikuläre Ejektionsfraktion bestimmt werden, die als wichtiger Prädiktor für die Mortalität der Herzinsuffizienz gilt (Likoff et al. 1987).

Die Lungenfunktionsprüfung gibt Hinweise auf eine pulmonale Stauung und besitzt klinische Bedeutung als Verlaufs- und Therapiekontrolle der Herzinsuffizienz (Gehlbach und Geppert 2004). Dabei werden alle gemessenen Parameter als Prozent bezogen auf einen Sollwert angegeben, der sich durch das Alter, die Größe und das Gewicht des Patienten ergibt.

Die Elektrokardiografie (EKG) zeigt typische Veränderungen bei Hypertrophie der Herzwand und kann durch die Darstellung von Herzrhythmusstörungen Hinweise auf die Genese einer Herzinsuffizienz geben (Deutsche Gesellschaft für Kardiologie 2005). 


\subsection{Cheyne-Stokes-Atmung und Herzinsuffizienz}

Bereits seit langer Zeit ist eine hohe Koinzidenz der Herzinsuffizienz mit der Cheyne-Stokes-Atmung bekannt. Verschiedene Studien konnten zeigen, dass die Prävalenz nächtlicher Atemstörungen in einem Kollektiv herzinsuffizienter Patienten bei bis zu 70 Prozent und darüber liegt (Sin et al. 1999, Schulz et al. 2007). Etwa 40 Prozent der Patienten mit Herzinsuffizienz zeigten hierbei in früheren Studien Atemstörungen zentraler Genese im Sinne einer CheyneStokes-Atmung (Javaheri et al. 1998, Lafranchi et al. 1999).

Wie unter 1.1.3. erläutert, gibt es eine ganze Reihe pathophysiologischer Mechanismen, die bei zugrunde liegender Herzinsuffizienz die Entstehung der Cheyne-Stokes-Atmung begünstigen. Außerdem existieren verschiedene Untersuchungen, die die Auswirkungen nächtlicher Schlafatemstörungen auf Entstehung und Verlauf einer Herzinsuffizienz beschreiben.

So führen hypoxische Episoden im Rahmen rezidivierender Apnoen zu einer Erhöhung des Sympathikotonus. Dies bedingt durch periphere Vasokonstriktion eine Erhöhung des systemischen Blutdrucks, außerdem wird ein positiv inotroper und chrontroper Effekt am Herzen erzeugt. Beides führt zu erhöhter Herzarbeit mit Steigerung des Sauerstoffbedarfs des Myokards und kann so zu einer Progredienz der Herzinsuffizienz im Sinne eines Circulus Vitiosus beitragen (Somers et al. 1993, Horner et al. 1994, Somers et al. 1995, Javaheri et al. 1995).

Durch die bei wiederkehrenden Hypoxien vermehrt im Stoffwechsel anfallenden freien Sauerstoffradikale wird im Verlauf inflammatorischer Kaskaden die Endothelfunktion geschädigt und dadurch der systemische Blutdruck unabhängig von der Sympathikusaktivität weiter erhöht (Dyugovskaya et al. 2002, Vongpatanasin et al. 2007). Weiterhin verschlechtern rezidivierende Hypoxien die Glukosetoleranz und erhöhen die Insulinresitenz peripherer Gewebe, so dass durch nächtliche Schlafatemstörungen die Enstehung eines Diabetes mellitus begünstigt wird und sich ein vorbestehender Diabetes verschlechtern kann (Oltmanns et al. 2004, Spiegel et al. 1999). Durch die Verschlechterung des kardiovaskulären Riskoprofils begünstigen nächtliche 
Schlafatemstörungen die Entstehung einer koronaren Herzkrankheit (Bradley und Floras 2009), die wiederum den höchsten Risikofaktor für die Entstehung einer Herzinsuffizienz darstellt (Fox et al. 2001).

Mehrere Studien konnten bei herzinsuffizienten Patienten eine bis zu zweimal erhöhte Mortalität mit dem Auftreten der Cheyne-Stokes-Atmung korellieren (Ancoli-Israel et al. 1994, Hanly und Zuberi-Khokar 1996, Lafranchi et al. 1999). Andere Studien konnten diesen Zusammenhang jedoch nicht sicher herstellen (Andreas et al. 1996 b, Roebuck et al. 2004). Es ist deshalb bisher noch unklar, ob die Cheyne-Stokes-Atmung einen unabhängigen Riskofaktor bei Herzinsuffizienz oder nur ein Epiphänomen darstellt.

\subsection{Medikamentöse Therapie der chronischen Herzinsuffizienz}

\subsubsection{Allgemeines}

Durch die Entwicklung neuer medikamentöser Therapiekonzepte konnte eine Verbesserung der Prognose von Patienten mit eingeschränkter linksventrikulärer Ejektionsfraktion in einer Vielzahl von Studien nachgewiesen werden. Von besonderer Bedeutung sind hierbei Medikamente aus der Gruppe der Angiotensin-Koversions-Enzym-Hemmer (ACE-Hemmer), der B-Adrenozeptorantagonisten (B-Blocker) und der Diuretika. Bei ACE-HemmerUnverträglichkeit gelten Medikamente aus der Gruppe der Angiotensinrezeptorantagonisten (AT1-Antagonisten) als gleichwertige Alternative. Weitere zur Herzinsuffizienztherapie indizierte Medikamente stammen aus der Gruppe der Digitalisglykoside und der Aldosteronantagonisten. Die Leitlinien der Deutschen Gesellschaft für Kardiologie zur Therapie der chronischen Herzinsuffizienz sehen eine Stufentherapie vor, die sich an der klinischen Stadieneinteilung der Herzinsuffizienz der New York Heart Association orientiert (Deutsche Gesellschaft für Kardiologie 2005, NYHA 1964) (Tabelle A).

Die verschiedenen Medikamentengruppen werden im Weiteren kurz erläutert. 
Tabelle A: Therapie der chronischen Herzinsuffizienz nach den Leitlinien der Deutschen Gesellschaft für Kardiologie (Deutsche Gesellschaft für Kardiologie 2005) (in Klammern die Empfehlungsgrade)

\begin{tabular}{|c|c|c|c|c|}
\hline Medikament & NYHA I & NYHA II & NYHA III & NYHA IV \\
\hline ACE-Hemmer & Indiziert (IA) & Indiziert (IA) & Indiziert (IA) & Indiziert (IA) \\
\hline$\beta$-Blocker & $\begin{array}{c}\text { nach } \\
\text { Myokardinfarkt } \\
\text { und bei } \\
\text { Hypertonie (IB) }\end{array}$ & Indiziert (IA) & Indiziert (IA) & Indiziert (IA) \\
\hline Diuretika & $\begin{array}{l}\text { Thiazide bei } \\
\text { Hypertonie }\end{array}$ & $\begin{array}{c}\text { bei } \\
\text { Flüssigkeitsretention } \\
\text { oder ehemals } \\
\text { vorgelegener } \\
\text { Flüssigkeitsretention } \\
\text { (IA) }\end{array}$ & Indiziert (IA) & Indiziert (IA) \\
\hline $\begin{array}{l}\text { Aldosteron- } \\
\text { antagonisten }\end{array}$ & $\begin{array}{c}\text { nach } \\
\text { Myokardinfarkt } \\
\text { (IB) }\end{array}$ & $\begin{array}{c}\text { nach Myokardinfarkt } \\
\text { (IB) }\end{array}$ & Indiziert (IB) & Indiziert (IB) \\
\hline $\begin{array}{l}\text { Digitalis- } \\
\text { glykoside }\end{array}$ & $\begin{array}{l}\text { bei tachykardem } \\
\text { Vorhofflimmern } \\
\text { (IB) }\end{array}$ & $\begin{array}{c}\text { bei tachykardem } \\
\text { Vorhofflimmern (IB) } \\
\text { oder } \\
\text { im Sinusrhythmus } \\
\text { nach Besserung } \\
\text { schwerer } \\
\text { Symptomatik (IlaC) }\end{array}$ & $\begin{array}{l}\text { Indiziert } \\
\text { (IlaC) }\end{array}$ & $\begin{array}{l}\text { Indiziert } \\
\text { (IlaC) }\end{array}$ \\
\hline
\end{tabular}




\subsubsection{ACE-Hemmer und AT1-Antagonisten}

ACE-Hemmer binden an das aktive Zentrum des Angiotensin-KonversionsEnzyms und hemmen dadurch die Umwandlung von Angiotensin $I$ in Angiotensin II. Angiotensin II gilt als starker Vasokonstriktor, so dass ACEHemmer den Blutdruck und damit die Nachlast des Herzens senken. Zahlreiche Studien zeigten, dass die Einnahme eines ACE-Hemmers die Symptome der Herzinsuffizienz signifikant bessert und die Hospitalisationsrate senkt (TRACE Study Group 1995, CONSENSUS Trial Study Group 1987). Des Weiteren beeinflussen ACE-Hemmer bei stabiler Herzinsuffizienz Morbidität und Mortalität an kardiovaskulären Ereignissen günstig, die Überlebensrate nach Myokardinfarkt steigt signifikant (Pfeffer et al. 1992). Bei Patienten mit asymptomatischer Herzinsuffizienz senken ACE-Hemmer die Inzidenz einer symptomatischen Herzinsuffizienz (SOLVD Investigators 1999). Die kardioprotektiven Effekte der ACE-Hemmer treten hierbei unabhängig von der Behandlung mit anderen Medikamenten der Herzinsuffizienztherapie auf (Flather et al. 2000). Von Captopril ist bekannt, dass es die Schlafqualität und die Inzidenz von nächtlichen Atemereignissen bei Patienten mit Cheyne-StokesAtmung positiv beeinflusst (Walsh et al. 1995).

AT1-Antagonisten dämpfen kompetitiv die Wirkung des Angiotensin II am Rezeptor und besitzen dadurch ein ähnliches Wirkungsspektrum wie ACEHemmer. In mehreren Studien konnte nachgewiesen werden, dass die Einnahme eines AT1-Antagonisten bei chronischer Herzinsuffizienz in gleichem Maße die Morbiditäts- und Mortalitätsrate sowie die Zahl der Hospitalisierungen senkt wie die Medikation mit einem ACE-Hemmer (Pitt et al. 2000, Granger et al. 2003).

Wegen der positiven Effekte auf Symptomatik und Verlauf der Erkrankung sind ACE-Hemmer Mittel der ersten Wahl zur Therapie der Herzinsuffizienz in jedem Stadium. AT1-Antagonisten stellen die alternative Medikamentengruppe bei Patienten mit Herzinsuffizienz und ACE-Hemmer-Unverträglichkeit dar (Deutsche Gesellschaft für Kardiologie 2005, European Society of Cardiology Task Force 2001). 


\subsubsection{B-Adrenozeptorantagonisten}

B-Adrenozeptorantagonisten (B-Blocker) sind kompetitive Antagonisten an BRezeptoren und verhindern deren Stimulation. Dadurch wird der positiv inotrope und chronotrope Einfluss des Sympathikus auf das Herz abgeschwächt und der myokardiale Sauerstoffverbrauch gesenkt.

Bei Langzeiteinnahme zeigt sich durch die Ökonomisierung des Energiehaushaltes des Herzens eine Verbesserung der Ventrikelfunktion mit Steigerung der Ejektionsfraktion (CIBIS Investigators and Committees 1994). Sie senken die Mortalität an kardiovaskulären Ereignissen sowie die Reinfarktrate nach Myokardinfarkt unabhängig von der Einnahme anderer Medikamente der Herzinsuffizienztherapie (CAPRICORN Investigators 2001). Vor allem in Kombination mit ACE-Hemmern können B-Blocker die Progredienz einer Herzinsuffizienz deutlich verlangsamen (Hjalmarson et al. 2000). B-Blocker werden empfohlen bei Herzinsuffizienz ab dem Stadium NYHA II, nach Myokardinfarkt auch ab NYHA I (Deutsche Gesellschaft für Kardiologie 2005, European Society of Cardiology Task Force 2001).

\subsubsection{Diuretika}

Diuretika hemmen die Elektrolytresorption in den Nierentubuli. Durch osmotische Bindung von Wasser erfolgt eine verstärkte Diurese und eine Abnahme des intravaskulären Flüssigkeitsvolumens. Folge ist eine Senkung der Vor- und Nachlast und dadurch des Sauerstoffbedarfs des Myokards.

Diuretika sind Mittel der ersten Wahl bei allen herzinsuffizienten Patienten, die Symptome einer Flüssigkeitsretention zeigen, bei gleichzeitig bestehender arterieller Hypertonie auch ohne Zeichen einer Flüssigkeitsrestriktion (Deutsche Gesellschaft für Kardiologie 2005, European Society of Cardiology Task Force 2001). 


\subsubsection{Aldosteronantagonisten}

Aldosteronantagonisten verdrängen Aldosteron kompetitiv von dessen intrazellulärem Rezeptor in den distalen Tubuluszellen der Niere. Hierdurch wird die renale Ausscheidung von Natrium verstärkt sowie die Kaliumausscheidung gehemmt und eine zusätzliche diuretische Wirkung entfaltet.

Bei Patienten mit einer Herzinsuffizienz nach stattgehabtem Myokardinfarkt senken Aldosteronantagonisten die Hospitalisationsrate sowie die Wahrscheinlichkeit, an einem plötzlichen Herztod zu sterben (Pitt et al. 2003). Weiterhin bessern Aldosteronantagonisten bei Patienten mit schwerer Herzinsuffizienz in den Stadien NYHA III und IV vor allem in Kombination mit einem ACE-Hemmer und einem B-Blocker die klinische Symptomatik und die Prognose des Patienten (Pitt et al. 1999).

Aldosteronantagonisten sind zur Therapie der chronischen Herzinsuffizienz ab dem NYHA-Stadium III, nach Myokardinfarkt bereits ab dem Stadium NYHA I indiziert (Deutsche Gesellschaft für Kardiologie 2005).

\subsubsection{Digitalisglykoside}

Digitalisglykoside steigern über eine Hemmung der Natrium-Kalium-ATPase die Kalziumkonzentration in der Herzmuskelzelle und wirken hierdurch positiv inotrop. Zusätzlich wird am Sinus- und AV-Knoten eine negativ chronotrope und dromotope Wirkung entfaltet.

Herzglykoside sind vor allem zur Frequenzkontrolle bei tachykard übergeleitetem Vorhofflimmern indiziert. Bei Patienten mit Sinusrhythmus und verminderter linksventrikulärer Ejektionsfraktion können Herzglykoside vor allem in Kombination mit ACE-Hemmern und B-Blockern die Symptomatik einer Herzinsuffizienz mildern und die Hospitalisationsrate aufgrund der Erkrankung senken (Digitalis Investigation Group 1997). In einer retrospektiven Studie konnte ein Überlebensvorteil bei herzinsuffizienten Männern mit einem niedrigen Serumspiegel nachgewiesen werden (Rathore et al. 2003). 
Die Leitlinien zur Herzinsuffizienzherapie sehen Herzglykoside ab dem Stadium NYHA III vor, bei bestehendem tachykard übergeleitetem Vorhofflimmern bereits ab dem Stadium NYHA I (Deutsche Gesellschaft für Kardiologie 2005).

\subsection{Fragestellung}

Javaheri et al. stellten fest, dass die Prävalenz signifikanter schlafbezogener Atemstörungen in einem Kollektiv chronisch herzinsuffizienter Patienten bei 51 Prozent liegt, wobei 40 Prozent der untersuchten Patienten zentrale Schlafatemstörungen im Sinne einer Cheyne-Stokes-Atmung zeigten (Javaheri et al. 1998). Eine andere Studie ergab eine Prävalenz schlafbezogener Atemsörungen von bis zu 72 Prozent, abhängig von der Anzahl der Atemereignisse, die zur Definition signifikanter schlafbezogener Atemstörungen zugrunde gelegt wurde. Hierbei zeigten 33 Prozent der Patienten des Gesamtkollektivs zentrale Atemstörungen (Sin et al. 1999). In neuerer Zeit durchgeführte Studien ergaben Prävalenzen schlafbezogener Atemstörungen zwischen 66 und 71 Prozent mit einem Anteil von Patienten mit zentral generierten Atemstörungen von bis zu 55 Prozent (Lafranchi et al. 2003, Ferrier et al. 2005, Schulz et al. 2007).

In keiner der genannten Studien wurde eine leitliniengerechte medikamentöse Herzinsuffizienztherapie als Einschlusskriterium vorausgesetzt. Die vorliegende Studie sollte deshalb nun untersuchen, ob die Einführung leitliniengerechter medikamentöser Therapiekonzepte die Prävalenz der Cheyne-Stokes-Atmung beeinflusst hat. 


\section{Methodik}

\subsection{Patientenkollektiv}

\subsubsection{Einschlusskriterien}

Das Patientenkollektiv setzte sich aus 50 konsekutiven Patienten der Abteilung Kardiologie und Pneumologie der Universitätsklinik Göttingen zusammen. Dabei kamen sowohl ambulante als auch stationäre Patienten in Frage. Die Patienten mussten zwischen 18 und 80 Jahren alt sein, wobei Patienten beiderlei Geschlechts in Frage kamen. Alle Patienten mussten sich zum Zeitpunkt der Untersuchung im NYHA-Stadium II oder III und in einem stabilen kardiopulmonalen Zustand befinden. Jedoch musste mindestens ein Ereignis einer kardialen Dekompensation anamnestisch eruierbar sein. Die echokardiografisch bestimmte linksventrikuläre Ejektionsfraktion durfte maximal 35 Prozent betragen. Alle Patienten mussten mit einem B-Blocker, einem ACEHemmer (bei Unverträglichkeit alternativ auch einem AT1-Antagonisten) und einem Diuretikum therapiert sein.

\subsubsection{Ausschlusskriterien}

Keiner der Patienten durfte Zeichen einer kardialen Dekompensation zeigen (wie z.B. Unterschenkelödeme oder Rasselgeräusche bei Auskultation über der Lunge). Ebenso durfte in den letzten 6 Monaten kein Herzinfarkt stattgefunden haben. Eine signifikante obstruktive Ventilationsstörung, definiert als forciertes exspiratorisches Lungenvolumen pro Sekunde dividiert durch die Vitalkapazität $<65$ Prozent vom Sollwert, führte ebenfalls zur Nichtberücksichtigung des Patienten. Weiterhin nicht teilnehmen durften Patientinnen in der Schwangerschaft oder Stillperiode, Patienten mit einer bekannten Suchterkrankung und Patienten mit relevanten Erkrankungen im HNO-Bereich. 
Bei Erfüllen der Einschlusskriterien und Fehlen von Ausschlusskriterien erfolgte die Auswahl unabhängig von allen anderen Faktoren wie z.B. Übergewicht, Müdigkeit, Schnarchen etc.

\subsection{Ablauf der Untersuchung}

Die Untersuchung fand in Form einer klinischen Studie im Zeitraum von Januar bis Dezember 2003 statt. Zu Beginn der Untersuchung wurde bei allen Patienten eine echokardiografische Bestimmung der linksventrikulären Ejektionsfraktion vorgenommen. Betrug diese $\leq 35$ Prozent, wurde die Einteilung des NYHA-Stadiums vorgenommen und die Patienten in Hinblick auf Ein- und Ausschlusskriterien ausführlich befragt. Zusätzlich wurde von allen Patienten im Vorfeld der polysomnografischen Untersuchung ein EKG angefertigt sowie eine Lungenfunktionsprüfung durchgeführt. Bei Erfüllen der Einschlusskriterien und Fehlen von Ausschlusskriterien wurden die Patienten mündlich und schriftlich über Zweck, Ziel und mögliche Risiken der Studie aufgeklärt und gaben auf einem gesonderten Blatt mit ihrer Unterschrift ihr Einverständnis zur Teilnahme an der Studie. Nach Einschluss der Patienten wurden Größe, Gewicht, alle zum Untersuchungszeitpunkt eingenommenen Medikamente und das kardiovaskuläre Risikoprofil (Vorhandensein einer arteriellen Hypertonie, eines Diabetes mellitus oder einer Hyperlipoproteinämie, Nikotinabusus) erfasst. Daraufhin wurden die Patienten für eine Nacht einer polysomnografischen Untersuchung im kardiologischen Schlaflabor der Universitätsklinik Göttingen unterzogen.

Die Ethikkommission der Universitätsklinik Göttingen hatte der Durchführung der Studie zugestimmt. 


\subsection{Polysomnografische Untersuchung}

Die polysomnografische Untersuchung für die Dauer einer Nacht fand im kardiologischen Schlaflabor der Universitätsklinik Göttingen statt. Hierfür wurden die Patienten vor dem Schlafengehen zu ihrer gewohnten Schlafenszeit nach alkoholischer Reinigung der betreffenden Körperstellen mit den nötigen Einmalklebeelektroden versehen (Abbildung 5). Die Ableitung des EEG erfolgte hierbei an den Stellen C3A2 und C4A1 des internationalen 10-20-Systems mit der Frequenzfiltereinstellung 0,5-30 Hz. Des Weiteren wurden zwei Elektroden zur Aufzeichnung eines EOG sowie eine Referenzelektrode auf der Glabella angebracht. Die Frequenzfiltereinstellung betrug hier $0,5-15 \mathrm{~Hz}$. Ein Oberflächen-EMG der Mundbodenmuskulatur sowie der lateralen Unterschenkelmuskulatur wurde mit einer Bandbreite des Filters von $3-100 \mathrm{~Hz}$ abgeleitet. Die Aufzeichnung der thorakalen und abdominellen Atemexkursionen erfolgte über zwei Druckmessaufnehmer. Der Atemfluss über der Nase wurde mit einem Thermistor registriert. Die pulsoxymetrische Aufzeichnung der Sauerstoffsättigung erfolgte über einen Clip-Sensor an einer Fingerspitze. Schließlich erfolgte die Ableitung eines Drei-Kanal-EKGs.

Die Aufnahme der nächtlichen Messdaten erfolgte mit dem System ALICE 4 der Firma Heinen \& Löwenstein. Die Darstellung der Aufzeichnung erfolgte über die gesamte Nacht in Epochen zu je 30 Sekunden auf Monitorbreite. Die Ableitungsqualität wurde, wenn nötig, korrigiert. Zum Zwecke der besseren Darstellung erfolgte während der Aufzeichnung eine ständige Anpassung der Signalverstärkung. 
Abbildung 5: Darstellung der Befestigungspunkte der Kopfelektroden. Nicht abgebildet ist die Referenzelektrode auf der Glabella (aus Rechtschaffen und Kales 1968 (Seite 19) mit freundlicher Genehmigung des ecomed-Verlages Landsberg).

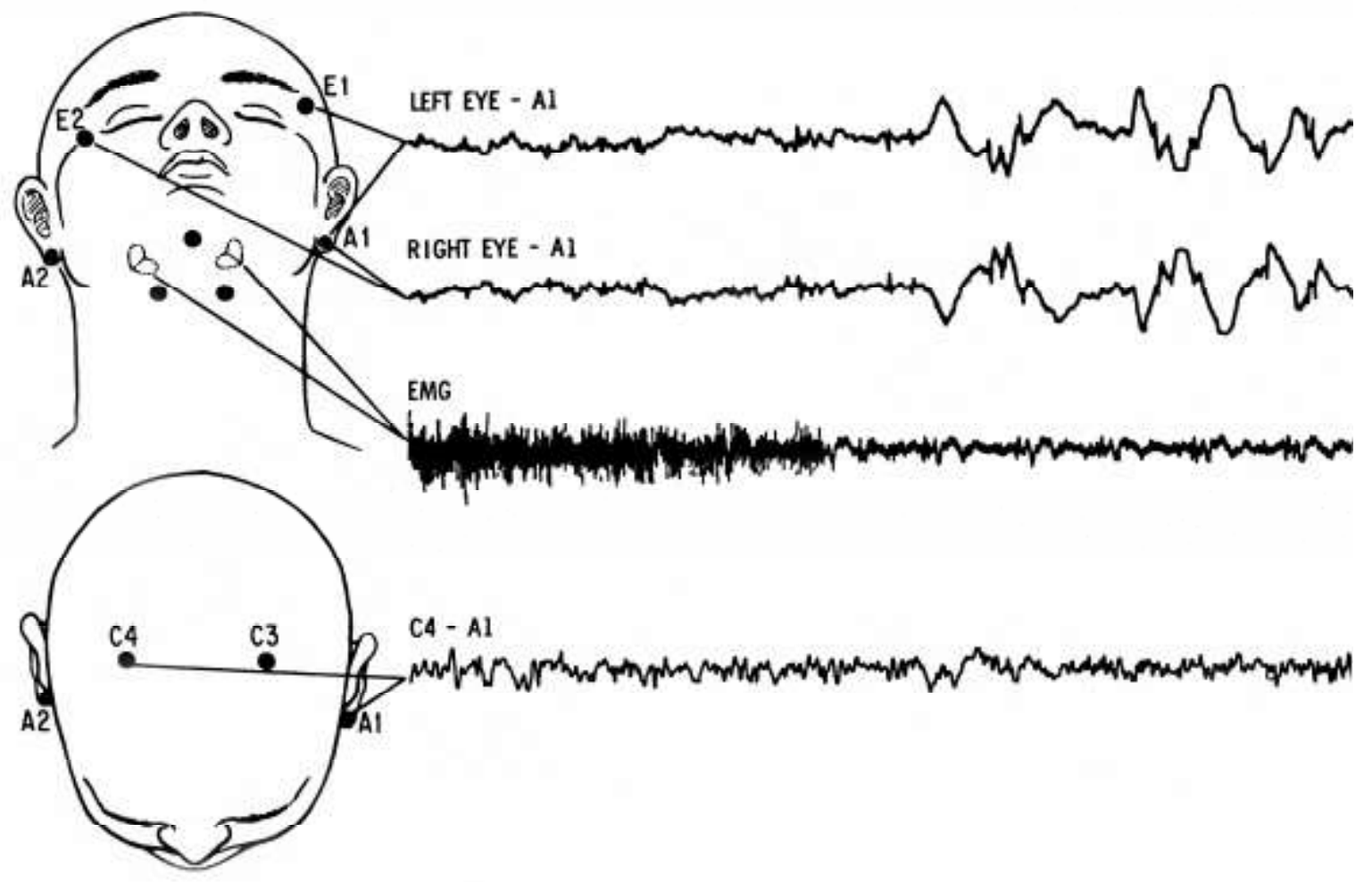




\subsection{Auswertung}

\subsubsection{Schlafstadien}

Die Auswertung der Schlafstadien erfolgte manuell in 30-Sekunden-Epochen nach den Kriterien von Rechtschaffen und Kales (Rechtschaffen und Kales (1968) (siehe Kapitel 1.1.4.).

Als Arousel wurde eine abrupte Veränderung der EEG-Frequenz während des Schlafes über drei oder mehr Sekunden mit einer Wellenfrequenz von mehr als $16 \mathrm{~Hz}$ definiert, die mit (während des REM Schlafes) oder ohne (während der Schlafstadien 1 bis 4) Veränderungen der EMG-Ableitung einhergeht (ASDA Task Force 1992).

\subsubsection{Atmung}

Als Hypopnoe wurde eine Abnahme des Atemflusses über der Nase um mehr als 50 Prozent für eine Dauer von mindestens zehn Sekunden bezeichnet. Ebenfalls als Hypopnoe wurde ein Ereignis gewertet, bei dem der Atemfluss über der Nase über zehn Sekunden um weniger als 50 Prozent abfällt, wenn diese Abnahme mit einem Abfall der kapillären Sauerstoffsättigung um mehr als drei Prozent oder einem Arousal assoziiert ist. Die Zeitdauer der Entsättigung war hierbei unerheblich.

Bei einer Apnoe sistierte der Atemfluss über der Nase für mindestens zehn Sekunden. Sie galt als zentrale Apnoe, wenn zusätzlich keine Atemexkursionen über Thorax und Abdomen ableitbar sind. Bei erhaltenen Atemexkursionen von Thorax und Abdomen wurde das Atemereignis als obstruktive Apnoe gewertet. Als Grenzwert zur Defintion signifikanter nächtlicher Atemstörungen wurde ein Apnoe-Hypopnoe-Index von 15/h festgelegt. 


\subsubsection{Statistik}

Alle Ergebnisse wurden bei Normalverteilung als Mittelwert \pm Standardabweichung angegeben. Bei nicht normaler Verteilung wurden die Werte als Median (oberes/unteres Quartil) angegeben. Für den Vergleich verschiedener Patientengruppen wurde bei Normalverteilung der Student-t-Test für unpaarige Stichproben, bei nicht normaler Verteilung der U-Test nach Wilcoxon für unpaarige Stichproben angewandt. Bei allen Tests wurde jeweils zweiseitig geprüft. Alle Berechnungen wurden mit dem Programm SigmaStat der Firma Systat Software (Version 3.1) durchgeführt. Ein p-Wert $<0,05$ wurde dabei als statistisch signifikant angesehen. 


\section{Ergebnisse}

\subsection{Gesamtes Patientenkollektiv}

\subsubsection{Allgemeine Patientendaten, Echokardiografie, EKG und Lungenfunktion}

In der Studie wurden insgesamt 50 Patienten untersucht, wovon 43 männlich und sieben weiblich waren. Das durchschnittliche Alter betrug $63 \pm 12$ Jahre. Der durchschnittliche Body-Mass-Index (BMI) lag bei 26,6 $\pm 4,3 \mathrm{~kg} / \mathrm{m}^{2}$ bei einer Körpergröße von $175 \pm 10 \mathrm{~cm}$ und einem Körpergewicht von 82 (71/88) kg. Hinsichtlich der Pathogenese der Herzinsuffizienz war die ischämische Kardiomyopathie mit 26 betroffenen Patienten die häufigste Ursache, gefolgt von der dilatativen Kardiomyopathie bei 20 Patienten und der hypertrophen Kardiomyopathie bei vier Patienten. Bei 31 Patienten bestand eine arterielle Hypertonie, bei 30 Patienten eine Hyperlipoproteinämie, bei 14 Patienten ein Diabetes mellitus und bei 14 Patienten ein anamnestisch erfassbarer Nikotinabusus. Bei acht Patienten bestand keiner dieser Risikofaktoren, elf Patienten waren mit einem, 19 Patienten mit zweien, acht Patienten mit dreien und vier Patienten mit allen vieren dieser Risikofaktoren vorbelastet. Alle Patienten waren mit einem $\beta$-Blocker und einem Diuretikum therapiert. Dazu erhielten 41 Patienten einen ACE-Hemmer und neun Patienten einen AT1-Antagonisten. 37 Patienten nahmen ein Antikoagulans ein, 33 Patienten ein Statin, 18 Patienten einen Aldosteronantagonisten und zwölf Patienten ein Digitalispräparat.

Die durchschnittliche linksventrikuläre Ejektionsfraktion betrug 25 (22/32) Prozent bei einem linksventrikulären enddiastolischen Durchmesser von $66 \pm 8$ $\mathrm{mm}$ und einem Durchmesser des linken Vorhofes von $50 \pm 8 \mathrm{~mm}$. Die Septumdicke betrug $11 \pm 2 \mathrm{~mm}$ im Durchschnitt.

Zum Zeitpunkt der Untersuchung zeigte das EKG bei 39 Patienten einen Sinusrhythmus und bei elf Patienten eine Arrhythmia absoluta bei Vorhofflimmern. Bei 28 Patienten fanden sich Zeichen eines Schenkelblocks. 
Die Lungenfunktionsuntersuchung zeigte durchschnittlich eine totale Lungenkapazität von $86 \pm 68$ Prozent bei einer Vitalkapazität von $74 \pm 19$ Prozent. Der Anteil der Einsekundenkapazität an der Vitalkapazität (TiffeneauTest) betrug $102 \pm 16$ Prozent, der Anteil des Residualvolumens an der totalen Lungenkapazität $118 \pm 28$ Prozent im Durchschnitt.

\subsubsection{Polysomnografische Untersuchung}

Die durchschnittliche Gesamtschlafzeit (Total Sleep Time) betrug 327 (254/363) Minuten. Die Dauer, welche die Probanden im Stadium REM verbrachten, betrug $51 \pm 28$ Minuten gegenüber einer NREM-Dauer von $271 \pm 65$ Minuten. Das Stadium NREM verbrachten die Probanden zu 94 (88/98) Prozent in den Leichtschlafstadien S1 und S2 und zu 6 (2/13) Prozent in den Tiefschlafstadien S3 und S4. Pro Stunde Schlaf traten durchschnittlich 14 (6/30) Atemereignisse auf mit durchschnittlich 4 (0/13) Apnoen und 6 (3/12) Hypopnoen. Dabei lag die durchschnittliche Sauerstoffsättigung des Blutes bei 91 (88/92) Prozent mit einer minimalen Sauerstoffsättigung von 84 (78/87) Prozent. Der Arousalindex, also die durchschnittliche Anzahl von Aufwachreaktionen pro Stunde Schlaf, betrug 26 (17/32).

\subsubsection{Prävalenz der Cheyne-Stokes-Atmung}

23 Patienten zeigten signifikante nächtliche Atemstörungen mit mehr als 15 Atemereignissen pro Stunde Schlaf, also einem AHI größer als 15/h. Bei 17 Patienten betrug der AHI mehr als 20/h, bei zehn Patienten fand sich ein AHI größer als 30/h.

Ein Patient bot das Bild eines obstruktiven Schlaf-Apnoe-Syndroms. Demgegenüber war bei 22 Patienten eine Cheyne-Stokes-Atmung nach Definition der AASM (American Academy of Sleep Medicine 2005) zu sehen. Dies entspricht einer Prävalenz von 44 Prozent. Der Anteil der Zeit mit CheyneStokes-Atmung an der gesamten Schlafzeit betrug bei diesen Patienten durchschnittlich 43 (23/73) Prozent. 


\subsection{Patientengruppen mit und ohne Cheyne-Stokes-Atmung}

\subsubsection{Patientengruppen}

In der Patientengruppe mit nächtlicher Cheyne-Stokes-Atmung (Gruppe CSA) befanden sich 21 männliche und ein weiblicher Patient gegenüber 22 männlichen und sechs weiblichen Patienten in der Patientengruppe ohne Cheyne-Stokes-Atmung (Gruppe NCSA). In beiden Gruppen war wie im Gesamtkollektiv die ischämische Kardiomyopathie die häufigste pathogenetische Ursache der Herzinsuffizienz, gefolgt von der dilatativen und der hypertrophen Form. Bezüglich des kardialen Risikoprofils unterschieden sich die beiden Patientengruppen nicht signifikant voneinander (Tabelle B). 
Tabelle B: Vergleich der anamnestisch erhobenen Daten der Patientengruppen mit und ohne Cheyne-Stokes-Atmung

\begin{tabular}{|c|c|c|c|}
\hline & $\begin{array}{c}\text { CSA } \\
(n=22)\end{array}$ & $\begin{array}{c}\text { NCSA } \\
(n=28)\end{array}$ & $\mathbf{p}$ \\
\hline \multicolumn{4}{|c|}{ Allgemeines } \\
\hline Alter (Jahre) & $62 \pm 14$ & $63 \pm 11$ & n.s. \\
\hline BMI $\left(\mathrm{kg} / \mathrm{m}^{2}\right)$ & $26 \pm 4$ & $27 \pm 5$ & n.s. \\
\hline Größe (cm) & $177 \pm 10$ & $174 \pm 9$ & n.s. \\
\hline Gewicht (kg) & $82(72 / 87)$ & $81(70 / 87)$ & n.s. \\
\hline \multicolumn{4}{|c|}{ Kardiale Risikofaktoren } \\
\hline Arterielle Hypertonie & 15 & 16 & n.s. \\
\hline Hyperlipoproteinämie & 14 & 16 & n.s. \\
\hline Diabetes mellitus & 6 & 8 & n.s. \\
\hline Nikotinabusus & 6 & 8 & n.s. \\
\hline \multicolumn{4}{|c|}{ Medikation } \\
\hline ACE-Hemmer & 18 & 23 & n.s. \\
\hline AT1-Anatagonist & 4 & 5 & n.s. \\
\hline Digitalispräparat & 4 & 8 & n.s. \\
\hline Aldosteronantagonist & 5 & 13 & n.s. \\
\hline Lipidsenker & 15 & 18 & n.s. \\
\hline Antikoagulans & 15 & 22 & n.s. \\
\hline
\end{tabular}

$\mathrm{CSA}=$ Patientengruppe mit Cheyne-Stokes-Atmung, NCSA = Patientengruppe ohne Cheyne-Stokes-Atmung, $\mathrm{BMI}=$ Body-Mass-Index, $\mathrm{ACE}=$ Angiotensin converting enzyme, AT1 = Angiotensinrezeptor 1, n.s.= nicht signifikant.

Angabe bei Normalverteilung als Mittelwert \pm Standardabweichung, bei nichtnormaler Verteilung als Median (unteres/oberes Quartil). 


\subsubsection{Echokardiografie, EKG und Lungenfunktion}

Ein Vergleich der in der Lungenfuktionsprüfung erhobenen Messwerte zeigte, dass die Vitalkapazität in der Gruppe CSA durchschnittlich $81 \pm 21$ Prozent vom Sollwert betrug, in der Gruppe NCSA hingegen nur $67 \pm 18$ vom Sollwert ( $p=0,009)$. Zudem zeigte sich in der Gruppe CSA ein signifikant höherer Wert für den Anteil der Einsekundenkapazität an der Vitalkapazität (104 \pm 15 vs. $100 \pm 16$ Prozent vom Sollwert in der Gruppe NCSA, $p<0,001)$. In der Gruppe NCSA betrug der Anteil des Residualvolumens an der totalen Lungenkapazität $127 \pm 25$ Prozent vom Sollwert gegenüber $107 \pm 28$ Prozent vom Sollwert in der Gruppe CSA. Dieser Unterschied war ebenfalls statistisch signifikant $(p=0,009)$. Bezüglich der in der Echokardiografie und dem EKG erhobenen Daten stellten sich keine Unterschiede zwischen den beiden Gruppen dar (Tabelle C). 
Tabelle C: Vergleich der Patientengruppen mit und ohne Cheyne-StokesAtmung bezüglich Echokardiografie, EKG und Lungenfunktion

\begin{tabular}{|c|c|c|c|}
\hline & $\begin{array}{c}\text { CSA } \\
(n=22)\end{array}$ & $\begin{array}{c}\text { NCSA } \\
(n=28)\end{array}$ & $\mathbf{p}$ \\
\hline \multicolumn{4}{|c|}{ Echokardiografie } \\
\hline $\mathrm{EF}(\%)$ & $25(21 / 32)$ & $25(22 / 32)$ & n.s. \\
\hline LVEDD (mm) & $65 \pm 7$ & $63 \pm 9$ & n.s. \\
\hline $\mathrm{LA}(\mathrm{mm})$ & $51 \pm 9$ & $49 \pm 7$ & n.s. \\
\hline Septumdicke $(\mathrm{mm})$ & $12 \pm 2$ & $11 \pm 2$ & n.s. \\
\hline \multicolumn{4}{|c|}{ EKG } \\
\hline Sinusrhythmus & 17 & 22 & n.s. \\
\hline Schenkelblock & 13 & 15 & n.s. \\
\hline \multicolumn{4}{|c|}{ Lungenfunktion } \\
\hline VC (\% Soll) & $81 \pm 21$ & $67 \pm 18$ & 0,009 \\
\hline TLC (\% Soll) & $88 \pm 73$ & $83 \pm 71$ & n.s. \\
\hline FEV1/VC (\% Soll) & $104 \pm 15$ & $100 \pm 16$ & $<0,001$ \\
\hline RV \% VC (\% Soll) & $107 \pm 28$ & $127 \pm 25$ & 0,009 \\
\hline
\end{tabular}

$\mathrm{CSA}=$ Patientengruppe mit Cheyne-Stokes-Atmung, NCSA= Patientengruppe ohne Cheyne-Stokes-Atmung, $E F=$ Ejektionsfraktion, $L V E D D=$ linksventrikulärer enddiastolischer Durchmesser, LA= Durchmesser linkes Atrium, VC= Vitalkapazität, FEV1 = forciertes exspiratorisches Volumen in einer Sekunde, $\mathrm{RV}=$ Residualvolumen, TLC= totale Lungenkapazität, n.s. = nicht signifikant.

Angabe bei Normalverteilung als Mittelwert \pm Standardabweichung, bei nichtnormaler Verteilung als Median (unteres/oberes Quartil). 


\subsubsection{Polysomnografische Daten}

Die beiden Gruppen unterscheiden sich erwartungsgemäß bezüglich des nächtlichen Atemprofils. So liegen alle Indices bezüglich der Atemauswertung (Apnoe-Hypopnoe-Index, Apnoe-Index, Hypopnoe-Index) in der Gruppe CSA weit über denen der Gruppe NCSA $(p<0,001)$. Zudem lag die minimale Sauerstoffsättigung in der Gruppe CSA signifikant niedriger als in der Gruppe NCSA ( $p=0,024)$. Bezüglich des übrigen Schlafprofils zeigten sich jedoch keine nennenswerten Unterschiede (Tabelle D). 
Tabelle D: Vergleich der Patientengruppen mit und ohne Cheyne-StokesAtmung bezüglich der polysomnografischen Daten

\begin{tabular}{c|c|c|c|} 
& $\begin{array}{c}\text { CSA } \\
(\mathbf{n = 2 2})\end{array}$ & $\begin{array}{c}\text { NCSA } \\
(\mathbf{n = 2 8})\end{array}$ & $\mathbf{p}$ \\
\hline TST (min) & $329(298 / 360)$ & $314(220 / 365)$ & n.s. \\
\hline REM (min) & $49 \pm 28$ & $53 \pm 29$ & n.s. \\
\hline NREM (min) & $285 \pm 43$ & $260 \pm 77$ & n.s. \\
\hline S1 + S2 \% NREM & $95(91 / 99)$ & $93(87 / 98)$ & n.s. \\
\hline S3 + S4 \% NREM & $5(1 / 9)$ & $7(0 / 14)$ & n.s. \\
\hline AHI (\#/h) & $26(19 / 40)$ & $6(4 / 9)$ & $<0,001$ \\
\hline Al (\#/h) & $14(8 / 36)$ & $1(0 / 3)$ & $<0,001$ \\
\hline HI (\#/h) & $12(7 / 15)$ & $4(2 / 6)$ & $<0,001$ \\
\hline Mittlere SaO2 (\%) & $91(87 / 92)$ & $91(88 / 93)$ & n.s. \\
\hline Minimale SaO2 (\%) & $81(77 / 86)$ & $86(83 / 89)$ & 0,024 \\
\hline Arousalindex (\#/h) & $25(19 / 32)$ & $27(16 / 32)$ & n.s.
\end{tabular}

$C S A=$ Patientengruppe mit Cheyne-Stokes-Atmung, NCSA $=$ Patientengruppe ohne Cheyne-Stokes-Atmung, TST $=$ Total Sleep Time, REM $=$ Rapid Eye Movement, NREM = Non-REM, S1-4= Schlafstadium 1-4, AHI= Apnoe-Hypopnoe-Index, Al= Apnoe-Index, $\mathrm{HI}=$ Hypopnoe-Index, $\mathrm{SaO} 2=$ kapilläre Sauerstoffsättigung, $n . \mathrm{s} .=$ nicht signifikant.

Angabe bei Normalverteilung als Mittelwert \pm Standardabweichung, bei nichtnormaler Verteilung als Median (unteres/oberes Quartil). 
3.3. Vergleich der polysomnografischen Daten der Patienten mit einer EF größer und kleiner als 25 Prozent

Die Messwerte der polysomnografischen Aufzeichnung ergeben eine höhere Anzahl von Aufwachreaktionen pro Stunde Schlaf in der Patientengruppe mit der höheren linksventrikulären Ejektionsfraktion $(p<0,04)$. Alle Messwerte bezüglich der Schlafstadienverteilung, der Atemereignisse sowie der Sauerstoffsättigung zeigten keine statistischen Unterscheidungsmerkmale zwischen den Gruppen (Tabelle E). 
Tabelle E: Vergleich der Patientengruppen mit EF $\leq 25$ und $>25$ Prozent bezüglich polysomnografischer Daten

\begin{tabular}{c|c|c|c|} 
& $\begin{array}{c}\mathrm{EF} \leq \mathbf{2 5} \% \\
\mathbf{( n = 2 8 )}\end{array}$ & $\begin{array}{c}\mathbf{E F}>\mathbf{2 5} \% \\
\mathbf{(} \mathbf{=} \mathbf{2 2})\end{array}$ & $\mathbf{p}$ \\
\hline TST (min) & $331(260 / 374)$ & $322(248 / 343)$ & n.s. \\
\hline REM (min) & $56 \pm 30$ & $44 \pm 25$ & n.s. \\
\hline NREM (min) & $277 \pm 68$ & $263 \pm 61$ & n.s. \\
\hline S1 + S2 \% NREM & $93(91 / 99)$ & $94(85 / 99)$ & n.s. \\
\hline S3 + S4 \% NREM & $7(1 / 9)$ & $6(1 / 15)$ & n.s. \\
\hline AHI (\#/h) & $12(4 / 28)$ & $13(6 / 22)$ & n.s. \\
\hline AI (\#/h) & $4(0 / 16)$ & $3(1 / 9)$ & n.s. \\
\hline HI (\#/h) & $7 \pm 5$ & $8 \pm 6$ & n.s. \\
\hline CSA & 18 & 15 & n.s. \\
\hline CSA \% TST & $43(18 / 73)$ & $19(12 / 25)$ & n.s. \\
\hline Mittlere SaO2 (\%) & $91(89 / 93)$ & $89(86 / 91)$ & n.s. \\
\hline Minimale SaO2 (\%) & $85(78 / 88)$ & $83(76 / 87)$ & n.s. \\
\hline Arousalindex (\#/h) & $23 \pm 11$ & $32 \pm 19$ & $<0,04$
\end{tabular}

$E F=$ linksventrikuläre Ejektionsfraktion, $T S T=$ Total Sleep Time, REM= Rapid Eye Movement, NREM= Non-REM, S1-4= Schlafstadium 1-4, AHI= Apnoe-HypopnoeIndex, $\mathrm{Al}=$ Apnoe-Index, $\mathrm{HI}=\mathrm{Hypopnoe}-$ Index, $\mathrm{CSA}=$ Cheyne-Stokes-Atmung, $\mathrm{SaO} 2=$ kapilläre Sauerstoffsättigung, n.s. $=$ nicht signifikant.

Angabe bei Normalverteilung als Mittelwert \pm Standardabweichung, bei nicht normaler Verteilung als Median (unteres/oberes Quartil). 


\section{Diskussion}

\subsection{Prävalenz der Cheyne-Stokes-Atmung}

Die Auswertung der vorliegenden Studie, die sich mit einem Kollektiv chronisch herzinsuffizienter Patienten in einer stabilen kardialen Verfassung beschäftigte, ergab eine Prävalenz der Cheyne-Stokes-Atmung von 44 Prozent und kommt damit zu einer vergleichbar hohen Prävalenz wie die historische Studie von Javaheri et al. (Javaheri et al. 1998). Vergleiche mit anderen, später durchgeführten Studien, die ebenfalls Patienten mit chronischer, kompensierter Herzinsuffizienz untersuchten, zeigen jedoch teilweise deutliche Unterschiede hinsichtlich der Prävalenz schlafbezogener Atemstörungen allgemein und der Cheyne-Stokes-Atmung im Besonderen (siehe Tabelle F). 
Tabelle F: Vergleich verschiedener Studien zur Prävalenz schlafbezogener Atemstörungen bei Herzinsuffizienz

\begin{tabular}{|c|c|c|c|c|c|c|}
\hline Autor & $\begin{array}{c}\text { Javaheri } \\
\text { et al. }\end{array}$ & $\begin{array}{l}\text { Sin } \\
\text { et al. }\end{array}$ & $\begin{array}{l}\text { Lafranchi } \\
\text { et al. }\end{array}$ & $\begin{array}{c}\text { Ferrier } \\
\text { et al. }\end{array}$ & $\begin{array}{l}\text { Schulz } \\
\text { et al. }\end{array}$ & $\begin{array}{l}\text { Aktuelle } \\
\text { Studie }\end{array}$ \\
\hline Jahr & 1998 & 1999 & 2003 & 2005 & 2007 & 2008 \\
\hline \multicolumn{7}{|c|}{ Studiendesign } \\
\hline $\begin{array}{l}\text { Definition } \\
\text { SBA }\end{array}$ & $\begin{array}{c}\mathrm{AHI} \\
\geq 15 / \mathrm{h}\end{array}$ & $\mathrm{AHI} \geq 10 / \mathrm{h}$ & $\mathrm{AHI} \geq 15 / \mathrm{h}$ & $\mathrm{AHI} \geq 10 / \mathrm{h}$ & $A H I \geq 10 / h$ & $\mathrm{AHI} \geq 15 / \mathrm{h}$ \\
\hline $\begin{array}{l}\text { Definition } \\
\text { CSA }\end{array}$ & $\begin{array}{c}\mathrm{OAHI} \leq \\
10 / \mathrm{h}\end{array}$ & $\begin{array}{c}>50 \% \\
\text { zentrale } \\
\text { Ereignisse }\end{array}$ & ----- & $\begin{array}{c}>50 \% \\
\text { zentrale } \\
\text { Ereignisse }\end{array}$ & $\begin{array}{c}50 \% \\
\text { zentrale } \\
\text { Ereignisse, } \\
\text { OAHI } \\
<10 / \mathrm{h}\end{array}$ & $\begin{array}{c}>50 \% \\
\text { zentrale } \\
\text { Ereignisse }\end{array}$ \\
\hline $\begin{array}{c}\text { EF (\%) } \\
\text { (Einschl.) }\end{array}$ & $<45$ & ----- & $\leq 45$ & $<45$ & $<40$ & $\leq 35$ \\
\hline \multicolumn{7}{|c|}{ Studienkollektiv } \\
\hline $\begin{array}{c}\text { Anzahl } \\
\text { Patienten }\end{array}$ & 81 & 450 & 47 & 53 & 203 & 50 \\
\hline $\begin{array}{l}\text { davon } \\
\text { männlich }\end{array}$ & $100 \%$ & $85 \%$ & $89 \%$ & $77 \%$ & $75 \%$ & $86 \%$ \\
\hline $\begin{array}{c}\text { Alter } \\
\text { (Jahre) }\end{array}$ & 64 & 60 & 59 & 60 & 65 & 63 \\
\hline $\begin{array}{c}\mathrm{BMl} \\
\left(\mathrm{kg} / \mathrm{m}^{2}\right)\end{array}$ & 28 & 29 & 26 & 28 & 28 & 27 \\
\hline $\mathrm{EF}(\%)$ & 25 & 27 & 27 & 34 & 28 & 25 \\
\hline $\begin{array}{c}\text { Prävalenz } \\
\text { SBA (\%) }\end{array}$ & 51 & 72 & 66 & 68 & 71 & 46 \\
\hline $\begin{array}{c}\text { Prävalenz } \\
\text { CSA (\%) }\end{array}$ & 40 & 33 & 55 & 15 & 28 & 44 \\
\hline
\end{tabular}

$\mathrm{SBA}=$ Schlafbezogene Atemstörungen, $\mathrm{AHI}=$ Apnoe-Hypopnoe-Index,

$\mathrm{OAHI}=$ Obstruktiver Apnoe-Hypopnoe-Index, CSA= Cheyne-Stokes-Atmung,

$\mathrm{EF}=$ Linksventrikuläre Ejektionsfraktion, $\mathrm{BMI}=$ Body-Mass-Index.

Angabe von Alter, BMI und EF im Bereich Studienkollektiv als Mittelwerte. 
Als Ursache für die verschieden hohen Prävalenzen schlafbezogener Atemstörungen in den verglichenen Studien kommen verschiedene Faktoren in Betracht.

Während für die Auswertung einer polysomnografischen Aufzeichnung und die Kriterien zur Analyse von nächtlichen Atemereignissen standardisierte und allgemein angewandte Richtlinien festgelegt wurden, werden häufig voneinander abweichende Defintionen für schlafbezogene Atemstörungen verwendet. Während Ancoli-Israel et al. 1994 noch einen Apnoe-Index von fünf Ereignissen pro Stunde als Grenzwert gewählt hatten (Ancoli-Israel et al. 1994), verwendeten spätere Studien für die Definition relevanter schlafbezogener Atemstörungen einen Apnoe-Hypopnoe-Index, wobei die Anzahl der geforderten Atemereignisse zur Definition bedeutsamer nächtlicher Schlafatemstörungen zwischen 10 und 15 pro Stunde variierte. Auch die Defintion der Cheyne-Stokes-Atmung unterscheidet sich in den verschiedenen Studien. Die vorliegende Studie benutzte als einzige der in Tabelle $\mathrm{F}$ angeführten Studien die Definition der AASM von 2005, die den Begriff CheyneStoke-Atmung synonym mit dem nächtlichen zentralen Schlafapnoesyndrom verwendet (zur Defintion siehe Kapitel 1.1.2). Zusätzlich wurde als Grenzwert zur Defintion signifikanter Atemstörungen ein AHI von 15 pro Stunde gewählt.

Drei Studien wählten einen AHI $\geq 10 / \mathrm{h}$ als Grenzwert (Sin et al. 1999, Ferrier et al. 2005, Schulz et al. 2007). Diese zeigten erwartungsgemäß eine höhere Gesamtprävalenz schlafbezogener Atemstörungen. Hierbei zeigte sich ein Überwiegen obstruktiver Atemstörungen, die Prävalenz der Cheyne-StokesAtmung fiel in diesen Studien hingegen deutlich geringer aus. Die drei Studien, die einen Grenzwert von 15 pro Stunde wählten (Javaheri et al. 1998, Lafranchi et al. 2003 und die hier vorliegende) ergaben niedrigere Gesamtprävalenzen, wobei sich jeweils ein hoher Anteil von Patienten mit zentralen Atemstörungen zeigte. Eine Ursache für diesen Zusammenhang könnte in der Beschaffenheit der jeweiligen Patientenkollektive liegen. Hinsichtlich der Geschlechterverteilung zeigt sich nämlich, dass in den Studien mit dem höchsten Anteil männlicher Patienten (Javaheri et al. 1998, Lafranchi et al. 2003 und die hier vorliegende Studie) zentrale Schlafatemstörungen häufiger vorkommen als in den übrigen 
Studien. Dies unterstützt die These, dass männliches Geschlecht als Risikofaktor nicht nur für schlafbezogene Atemstörungen insgesamt, sondern auch für die Entwicklung der Cheyne-Stokes-Atmung gelten kann.

Ob möglicherweise gemischte Apnoen, also obstruktive Atemstörungen, die in inrem Verlauf in zentralen Apnoen übergehen, in den Studien von Sin et al., Ferrier et al. und Schulz et al. (Sin et al. 1999, Ferrier et al. 2005, Schulz et al. 2007) als obstruktive Atemereignisse gewertet wurden und so eine hohe Prävalenz dieser Atemstörungen bedingt haben, geht aus den Studien nicht hervor und bleibt somit Spekulation. Die verglichenen Studienkollektive unterscheiden sich ausser in Bezug auf die Geschlechterverteilung hinsichtlich der übrigen klassischen Risikofaktoren für schlafbezogene Atemstörungen (hoher BMI und hohes Alter) nicht signifikant voneinander, so dass durch die demografischen Daten die hohe Prävalenz obstruktiver Atemstörungen nicht erklärt werden kann.

In der aktuell durchgeführten Studie wurden Patienten erst mit einer linksventrikulären Ejektionsfraktion von $\leq 35$ Prozent eingeschlossen. Da nach den gängigen pathophysiologischen Erklärungsmodellen eine schwerere Linksherzinsuffizienz die Enstehung einer Cheyne-Stokes-Atmung begünstigt, ist im aktuell untersuchten Patientenkollektiv also mit einer höheren Prävalenz zentraler Atemstörungen zu rechnen. Dieser Zusammenhang kann die niedrigere Prävalenz der Cheyne-Stokes-Atmung in der Studie von Ferrier et al. erklären, da hier der Mittelwert der linksventrikulären Ejektionsfraktion deutlich höher liegt als im Kollektiv der hier vorliegenden Studie. Die niedrigere Prävalenz in den Studien von Sin et al. (Sin et al. 1999) und Schulz et al. (Schulz et al. 2007) kann hierdurch jedoch nicht erklärt werden, da sich trotz verschiedener Einschlusskriterien die Mittelwerte der linksventrikulären Ejektionsfraktionen nicht wesentlich von der des aktuell untersuchten Kollektivs unterscheiden. Auch hinsichtlich des Body-Mass-Index und des Alters bestehen keine großen Unterschiede, obwohl beide Faktoren, wie erwähnt, als Risikofaktoren für die Entstehung schlafbezogener Atemstörungen und der Cheyne-Stokes-Atmung gelten. 
Der Großteil der Patienten aus der Studie bei Javaheri et al. befand sich zum Zeitpunkt der durchgeführten polysomnografischen Untersuchung in den Herzinsuffizienzstadien NYHA I und II (Javaheri et al. 1998), bei Sin et al. wurden Patienten in den NYHA-Stadien II-IV untersucht (Sin et al. 1999). Die hier vorliegende Studie befasste sich ausschließlich mit Patienten in den NYHAStadien II und III. Eine direkte Aktivierung der Dehnungsrezeptoren der Lunge bei pulmonaler Stauung führt vagal vermittelt zu einer Hyperventilation mit chronischer Hypokapnie, was beim Übergang vom Wachzustand in den Schlaf einen Abfall des $\mathrm{PCO}_{2}$ unter die Apnoeschwelle herbeiführt und somit nächtliche Atemstörungen begünstigt (Churchill und Cope 1929, Dempsey und Skatrud 1986, Naughton et al. 1993). Rezidivierende hypoxische Ereignisse, wie sie mutmaßlich häufiger bei Patienten höherer NYHA-Stadien vorkommen, führen zudem über eine Sensibilisierung der Messfühler an Aorta und Karotisgabel zu einer verstärkten Atemantwort auf Veränderungen der Atemgase und begünstigen somit ebenfalls nächtliche Atemstörungen (Dowell et al. 1971, Chapmann et al. 1988). Wenn man aufgrund des höheren Anteils an Patienten mit symptomatischer Herzinsuffizienz bei Sin et al. (Sin et al. 1999) und in der vorliegenden Studie auch von einem erhöhten Anteil von Patienten mit chronischer, kardial bedingter pulmonaler Stauung ausgeht, wäre eigentlich eine höhere Prävalenz der Cheyne-Stokes-Atmung im Vergleich zur Studie von Javaheri et al. (Javaheri et al. 1998) zu erwarten gewesen. Für die trotzdem ähnlich hohe Prävalenz zentraler Atemstörungen kommen verschiedene Ursachen in Frage. So zeigen sich vergleichbare Mittelwerte für die Risiokofaktoren linksventrikuläre Ejektionsfraktion, Alter und BMI. Während Javaheri et al. zur Defintion der Cheyne-Stokes-Atmung lediglich einen AHI $\geq 15 / \mathrm{h}$ bei einem obstruktiven AHI $\leq 10 / \mathrm{h}$ forderten (Javaheri et al. 1998), hielt sich die vorliegende Studie zusätzlich zu einem AHI $\geq 15 / h$ als Grenzwert an die Defintion der AASM, die weiterhin eine hohe Tagesmüdigkeit sowie Sauerstoffentsättigungen und Arousel in Zusammenhang mit den Atemereignissen fordert. Diese strengere Definiton mag dazu geführt haben, dass trotz des höheren Anteils von Patienten mit symptomatischer Herzinsuffizienz weniger Patienten die Kriterien einer Cheyne-Stokes-Atmung erfüllt haben als in der Studie von Javaheri et al. (Javaheri et al. 1998). 
Schließlich lässt der höhere Anteil männlicher Patienten in der Studie von Javaheri et al. (Javaheri et al. 1998) einen höheren Anteil nächtlicher Atemstörungen erwarten.

Bei Sin et al. wurden Patienten noch bis in das Stadium NYHA IV in die Studie eingeschlossen (Sin et al. 1999), zeigten also zum Untersuchungszeitpunkt teilweise Zeichen einer kardialen Dekompensation. Die Prävalenz schlafbezogener Atemstörungen in einem Kollektiv kardial dekompensierter Patienten ist höher ist als in einem Kollektiv von Patienten mit stabiler chronischer Herzinsuffizienz (Dark et al. 1987, Tremel et al. 1999, Mared et al. 2004), so dass der mit 38 Prozent hohe Anteil von Patienten mit Beschwerden in Ruhe und bei nur leichter Belastung, zusätzlich zum niedriger gewählten AHI als Grenzwert, eine Ursache für die in der Studie von Sin et al. (Sin et al. 1999) deutlich höher ermittelte Prävalenz schlafbezogener Atemstörungen sein könnte. In den übrigen Studien wurden keine NYHA-Stadien erfasst (Ferrier et al. 2005, Schulz et al. 2007) oder ausschließlich asymptomatische Patienten untersucht (Lafranchi et al. 2003) so dass Vergleiche der Prävalenzen schlafbezogener Atemstörungen in Bezug auf klinische Parameter hier nicht gezogen werden können.

Vergleicht man die hier vorliegenden Studienergebnisse mit denen der Studie von Schulz et al. von 2007, zeigt sich bei Schulz et al. eine deutlich höhere Prävalenz nächlicher Atemstörungen als im hier untersuchten Kollektiv, wobei ein hoher Anteil von Patienten ein obstruktives Schlafapnoesyndrom aufwies bei sehr viel niedrigerem Anteil von Patienten mit zentralen Atemstörungen. Dies könnte erneut an der Defintion der Cheyne-Stokes-Atmung liegen. Von Schulz et al. wurde nämlich neben einem Überwiegen zentraler Atemstörungen ein obstruktiver AHI von unter 10 pro Stunde gefordert (Schulz et al. 2007). Da sich im dort untersuchten Kollektiv ein hoher Anteil von Patienten mit obstruktiven Atemstörungen zeigte, könnte dies die Ursache dafür sein, dass weniger Patienten die Kriterien zur Defintion der Cheyne-Stokes-Atmung erfüllten. Warum die Gesamtprävalenz schlafbezogener Atemstörungen jedoch deutlich höher ausgefallen ist, bleibt unklar, da sich außer der Patientenzahl die Kollektive nicht signifikant unterscheiden und der Anteil weiblicher Patienten in der Studie von Schulz et al. sogar höher ist als in der hier durchgeführten Studie (Schulz et al. 2007). 


\subsection{Einfluss der medikamentösen Herzinsuffizienztherapie}

Die meisten Vergleichsstudien zur Prävalenz schlafbezogener Atemstörungen untersuchten die Patienten ohne eine standardisierte, den Leitlinien entsprechenden medikamentöse Herzinsuffizienztherapie. Es wurden verschiedene Medikamentengruppen in der Therapie der chronischen Herzinsuffizienz etabliert, die zu einem nachweislichen Mortalitätsrückgang geführt haben. Die aktuellen Leitlinien der Deutschen Gesellschaft für Kardiologie zur Therapie der chronischen Herzinsuffizienz sehen ein Stufenschema vor, das sich an der Stadieneinteilung der New York Heart Association orientiert (NYHA 1964). Da sich alle in der vorliegenden Studie untersuchten Patienten im Stadien NYHA II oder III befanden und mindestens ein Ereignis einer kardialen Dekompensation eruierbar sein musste, mussten alle Patienten leitliniengemäß mit einem ACE-Hemmer bzw. alternativ einem AT1-Antagonisten, einem B-Blocker und einem Diuretikum therapiert sein. Des Weiteren erhielten 12 Patienten ein Digitalispräparat und 18 Patienten einen Aldosteronantagonisten. Ziel der vorliegenden Untersuchung war es zu prüfen, ob es hierunter zu einer Änderung der Prävalenz der Cheyne-Stokes-Atmung gekommen ist.

Campbell et al. stellten bereits 1981 fest, dass nach Gabe eines Betablockers durch Verminderung der Chemozeptorsensitivität die Atemantwort auf einen erhöhten Kohlendioxidpartialdruck vermindert wird (Campbell et al. 1981). Dementsprechend konnten Studien zeigen, dass herzinsuffiziente Patienten unter einer Betablockertherapie signifikant weniger zentrale Atemstörungen aufweisen als Patienten ohne diese Therapie (Köhnlein und Welte 2007, Tamura et al. 2007). Als Ursache hierfür wird neben einer Verminderung der Zirkulationszeit eine Abnahme der pulmonalvenösen Stauung diskutiert (Schulz et al. 2007).

Obwohl weiterhin gezeigt werden konnte, dass zumindest nach Einnahme von Captopril eine Reduktion nächtlicher Atemereignisse und eine Verbesserung der Schlafqualität stattfindet (Walsh et al. 1995), war die Prävalenz der CheyneStokes-Atmung im hier untersuchten Kollektiv im Vergleich zu anderen 
Studienkollektiven ohne standardisierte ACE-Hemmer- und BetablockerEinnahme ähnlich hoch oder höher, wenn man von der Studie von Lafranchi et al. absieht. In Zusammenschau der im Rahmen der vorliegenden Studie erhobenen Daten ergeben sich somit keine Hinweise darauf, dass eine leitliniengerechte Herzinsuffizienztherapie zu einem signifikanten Rückgang der Prävalenz der Cheyne-Stokes-Atmung in einem Kollektiv chronisch herzinsuffizienzter Patienten führt. Möglicherweise lässt sich durch Untersuchung verschiedener Kontrollgruppen mit definierten Unterschieden im medikamentösen Therapiekonzept Aufschluss darüber gewinnen, welche Medikamentengruppen einen günstigen Einfluss auf Entstehung oder Ausprägung nächtlicher Atemstörungen haben. Solche Kontrollgruppen wurden jedoch im Rahmen der Studie nicht untersucht. Ebenso wurden der Zeitpunkt der Einleitung der leitliniengerechten Medikation nicht erfasst und die hier untersuchten Patienten keiner polysomnografischen Kontrolluntersuchung nach einem definierten Behandlungsintervall unterzogen, weshalb keine Aussagen über mögliche Langzeittherapieeffekte möglich sind. Es könnte sein, dass eine Beeinflussung der nächtlichen Atemstörungen erst unter einer medikamentösen Langzeittherapie auftritt und demnach durch das hier gewählte Studiendesign nicht erfasst wurde. Sollten zukünftige klinische Studien die hier erhobenen Daten bestätigen, scheint ein wichtiger therapeutischer Ansatz zur weiteren Senkung der Mortalität bei Herzinsuffizienz in der weiteren Erforschung einer wirksamen Therapie nächtlicher Atemstörungen zu liegen. 


\subsection{Weitere Erkenntnisse}

Sin et al. beschrieben 1999 ein Alter von über 60 Jahren als Risikofaktor für das Vorhandensein einer Cheyne-Stokes-Atmung (Sin et al. 1999). Mared et al. beschrieben ebenfalls eine zunehmende Prävalenz mit ansteigendem Lebensalter (Mared et al. 2004). Die vorliegende Studie konnte keinen solchen Zusammenhang feststellen und geht damit konform mit einer anderen Studie zu diesem Thema (Mortara et al. 1999). Ebenso konnte kein Zusammenhang zwischen der Geschlechterverteilung der beiden Gruppen mit und ohne Cheyne-Stokes-Atmung festgestellt werden, so dass sich nicht sicher bestätigen ließ, dass männliches Geschlecht als Risikofaktor für die Entstehung schlafbezogener Atemstörungen gelten kann. Hierbei ist allerdings zu berücksichtigen, dass der Anteil weiblicher Patienten am Gesamtkollektiv nur 14 Prozent betrug und somit keine statistisch belastbare Aussage über eine geschlechtsspezifische Prävalenz möglich ist.

In der hier durchgeführten Studie wurden 50 Patienten untersucht, was im Vergleich zu anderen epidemiologischen Studien einem eher kleinen Kollektiv entspricht. Durch Untersuchung größerer Patientenkollektive könnten genauere Aussagen zur Prävalenz der Cheyne-Stokes-Atmung unter leitliniengerechter medikamentöser Herzinsuffizienztherapie möglich werden. Kontrollgruppen bezüglich verschiedenster Faktoren, etwa des kardiovaskulären Risikoprofils oder verschiedener medikamentöser Therapieregime, könnten positive und negative Einflüsse auf die Entstehung nächtlicher Schlafatemstörungen und insbesondere der Cheyne-Stokes-Atmung genauer eruieren und so eventuell zu einer weiteren Senkung von Morbidität und Mortalität bei Herzinsuffizienz beitragen.

Signifikante Unterschiede zwischen den beiden Gruppen mit und ohne CheyneStokes-Atmung zeigten sich bezüglich der Lungenfunktion. So zeigte die Patientengruppe ohne Cheyne-Stokes-Atmung bei vergleichbaren Daten für die totale Lungenkapazität eine signifikant niedrigere Vitalkapazität, eine niedrigere Einsekundenkapazität bezogen auf die Vitalkapazität sowie einen höheren 
Anteil des Residualvolumen an der totalen Lungenkapazität. Es ist also in der Patientengruppe ohne Cheyne-Stokes-Atmung von einem höheren Anteil von Patienten mit obstruktiver, restriktiver oder kombinierter Ventilationsstörung auszugehen. Ein hohes Residualvolumen erhöht den $\mathrm{CO}_{2}$-Speicher des Organismus und stabilisiert so nach den gängigen pathophysiologischen Erklärungsmodellen für die Entstehung zentraler Atemstörungen den Regelkreis der Atmung. Wenn man aufgrund des höheren Anteil von Patienten mit Ventilationsstörungen auf einen größeren Gesamt-CO2-Speicher schließt, könnte dies eine Erklärung für die niederigere Prävalenz zentraler Atemstörungen in dieser Gruppe sein. Da eine Erhebung der arteriellen oder gemischtvenösen $\mathrm{CO}_{2}$-Werte in dieser Studie jedoch nicht durchgeführt wurde, ist diese These nicht zu beweisen und muss Vermutung bleiben. 


\section{Zusammenfassung}

Zur Erhebung der Prävalenz der Cheyne-Stokes-Atmung unter leitliniengerechter medikamentöser Herzinsuffizienztherapie wurden 50 Patienten mit chronischer Herzinsuffizienz und einer linksventrikulären Ejektionsfraktion von 35 Prozent oder weniger einer Polysomnografie unterzogen. Alle Patienten mussten sich zum Zeitpunkt der Untersuchung in einer stabilen kardialen Situation befinden und mit einer leitliniengerechten medikamentösen Herzinsuffizienztherapie behandelt sein. 44 Prozent der untersuchten Patienten zeigten eine nächtliche Cheyne-Stokes-Atmung nach den Kriterien der AASM (AASM 2005), so dass im Vergleich zu historischen Kollektiven ohne standardisierte Herzinsuffizienztherapie (Javaheri et al. 1998, Sin et al. 1999) kein wesentlicher Rückgang zentraler Atemstörungen unter leitliniengerechter Medikation zu verzeichnen ist.

Mit einer linksventrikulären Ejektionsfraktion von 35 Prozent als Einschlusskriterium liegt die vorliegende Studie niedriger als vorhergehende Untersuchungen, so dass im Hinblick auf die pathophysiologische Genese zentraler Atemstörungen die durchschnittlich schlechtere kardiale Funktion eine Ursache für die im Vergleich zu historischen Kollektiven erhöhte Prävalenz der Cheyne-Stokes-Atmung im aktuell untersuchten Kollektiv sein könnte. Durch das mit 50 untersuchten Patienten im Vergleich zu anderen Studien eher kleine Kollektiv wird die statistische Aussagekraft gemindert, ebenso sind aufgrund des niedrigen Anteils weiblicher Patienten keine belastbaren Aussagen über die Geschlechterverteilung nächtlicher Atemstörungen und die Prävalenz der Cheyne-Stokes-Atmung in der Gesamtpopulation herzinsuffizienter Patienten möglich. 


\section{Anhang}

\subsection{Tabellen}

Tabelle G: Anamnestische Daten, Echokardiografie, EKG, Lungenfunktion

\begin{tabular}{|c|c|c|c|c|c|c|c|c|c|}
\hline Pat. & $\mathbf{G}$ & Alter & BMI & HI & EF (\%) & LVEDD (mm) & SR & VC (\%Soll) & FEV1/VC (\%Soll) \\
\hline 1 & $\mathrm{~m}$ & 62 & 26,45 & $\overline{\mathrm{DCM}}$ & 35 & 52 & ja & 81 & 113 \\
\hline 2 & $\mathrm{~m}$ & 62 & 27,74 & HNOCM & 20 & 56 & nein & 44 & 133 \\
\hline 3 & $\mathrm{w}$ & 76 & 24,00 & ICM & 25 & 59 & ja & 99 & 118 \\
\hline 4 & $\mathrm{~m}$ & 76 & 24,57 & ICM & 31 & 54 & ja & 73 & 93 \\
\hline 5 & $\mathrm{~m}$ & 72 & 20,07 & DCM & 32 & 64 & $\mathrm{ja}$ & 125 & 90 \\
\hline 6 & $\mathrm{~m}$ & 57 & 24,69 & $\overline{\mathrm{DCM}}$ & 20 & 67 & ja & 83 & 94 \\
\hline 7 & $\mathrm{~m}$ & 81 & 26,99 & ICM & 35 & 65 & ja & 43 & 99 \\
\hline 8 & $\mathrm{~m}$ & 74 & 28,36 & $\overline{\mathrm{DCM}}$ & 20 & 78 & nein & 63 & 105 \\
\hline 9 & $\mathrm{~m}$ & 68 & 24,56 & $\mathrm{ICM}$ & 30 & 54 & $\mathrm{ja}$ & 93 & 105 \\
\hline 10 & $\mathrm{~m}$ & 79 & 26,42 & ICM & 35 & 64 & ja & 76 & 127 \\
\hline 11 & $\mathrm{~m}$ & 29 & 37,37 & DCM & 25 & 79 & ja & 71 & 86 \\
\hline 12 & $m$ & 44 & 20,07 & ICM & 22 & 61 & ja & 53 & 87 \\
\hline 13 & $\mathrm{~m}$ & 78 & 23,66 & ICM & 33 & 60 & ja & 94 & 118 \\
\hline 14 & $m$ & 74 & 23,18 & ICM & 35 & 72 & ja & 51 & 128 \\
\hline 15 & $\mathrm{~m}$ & 47 & 39,23 & HNOCM & 34 & 56 & nein & 85 & 104 \\
\hline 16 & $\mathrm{~m}$ & 54 & 25,39 & ICM & 30 & 64 & ja & 90 & 107 \\
\hline 17 & $\mathrm{~m}$ & 65 & 24,82 & HNOCM & 25 & 57 & nein & 73 & 102 \\
\hline 18 & $\mathrm{~m}$ & 62 & 27,19 & ICM & 33 & 70 & ja & 65 & 103 \\
\hline 19 & $\mathrm{~m}$ & 65 & 23,43 & ICM & 23 & 70 & ja & 108 & 93 \\
\hline 20 & $\mathrm{~m}$ & 65 & 26,80 & DCM & 25 & 65 & ja & 77 & 105 \\
\hline 21 & $w$ & 41 & 36,76 & $\overline{\mathrm{DCM}}$ & 20 & 67 & ja & 102 & 111 \\
\hline 22 & $\mathrm{~m}$ & 60 & 29,71 & ICM & 27 & 59 & ja & 85 & 112 \\
\hline 23 & $\mathrm{~m}$ & 66 & 21,67 & HNOCM & 25 & 50 & ja & 72 & 111 \\
\hline 24 & $\mathrm{~m}$ & 76 & 29,36 & DCM & 27 & 76 & nein & 65 & 106 \\
\hline 25 & $m$ & 75 & 24,66 & ICM & 25 & 68 & nein & 56 & 79 \\
\hline 26 & $w$ & 72 & 25,89 & ICM & 35 & 48 & ja & 64 & 107 \\
\hline 27 & $m$ & 64 & 24,28 & $\overline{\mathrm{DCM}}$ & 10 & 72 & nein & 69 & 116 \\
\hline 28 & $m$ & 49 & 28,70 & ICM & 20 & 43 & ja & 71 & 76 \\
\hline 29 & $\mathrm{~m}$ & 74 & 22,36 & ICM & 25 & 73 & ja & 73 & 102 \\
\hline 30 & $\mathrm{~m}$ & 62 & 28,72 & ICM & 33 & 63 & nein & 60 & 97 \\
\hline 31 & $\mathrm{~m}$ & 49 & 31,35 & DCM & 25 & 72 & ja & 86 & 110 \\
\hline 32 & $w$ & 60 & 34,00 & ICM & 9 & 57 & ja & 43 & 98 \\
\hline 33 & $\mathrm{~m}$ & 54 & 22,84 & ICM & 23 & 68 & ja & 64 & 103 \\
\hline 34 & $w$ & 78 & 22,79 & ICM & 34 & 50 & ja & 84 & 94 \\
\hline 35 & $\mathrm{~m}$ & 75 & 21,78 & ICM & 34 & 60 & nein & 69 & 70 \\
\hline 36 & $\mathrm{~m}$ & 74 & 26,13 & $\overline{\mathrm{DCM}}$ & 25 & 69 & ja & 50 & 92 \\
\hline 37 & $\mathrm{~m}$ & 56 & 28,04 & DCM & 25 & 57 & ja & 108 & 111 \\
\hline 38 & $\mathrm{~m}$ & 75 & 24,54 & ICM & 23 & 61 & ja & 38 & 104 \\
\hline 39 & $\mathrm{~m}$ & 47 & 31,79 & DCM & 25 & 67 & ja & 46 & 96 \\
\hline 40 & $\mathrm{~m}$ & 47 & 30,91 & DCM & 30 & 67 & ja & 73 & 102 \\
\hline 41 & $\mathrm{~m}$ & 35 & 30,61 & $\overline{\mathrm{DCM}}$ & 19 & 78 & ja & 61 & 107 \\
\hline 42 & $w$ & 58 & 20,59 & DCM & 25 & 63 & ja & 98 & 109 \\
\hline 43 & $\mathrm{~m}$ & 45 & 29,39 & DCM & 20 & 67 & ja & 73 & 102 \\
\hline 44 & $\mathrm{~m}$ & 54 & 26,83 & ICM & 28 & 62 & ja & 46 & 77 \\
\hline 45 & $\mathrm{~m}$ & 63 & 26,99 & DCM & 27 & 68 & $\mathrm{ja}$ & 78 & 130 \\
\hline 46 & $\mathrm{~m}$ & 58 & 23,70 & ICM & 20 & 67 & ja & 64 & 93 \\
\hline 47 & $m$ & 68 & 25,21 & $\overline{\mathrm{DCM}}$ & 19 & 78 & ja & 86 & 98 \\
\hline 48 & $\mathrm{~m}$ & 70 & 31,44 & $\overline{\mathrm{DCM}}$ & 35 & 57 & nein & 49 & 54 \\
\hline 49 & $\mathrm{~m}$ & 65 & 24,08 & ICM & 17 & 63 & nein & 90 & 99 \\
\hline 50 & $m$ & 65 & 20,76 & ICM & 30 & 70 & ja & 77 & 113 \\
\hline
\end{tabular}

$\mathrm{G}=$ Geschlecht, $\mathrm{BMI}=$ Body-Mass-Index, $\mathrm{HI}=$ Pathogenese der Herzinsuffizienz, DCM= dilatative Kardiomyopathie, ICM= ischämische Kardiomyopathie, $\mathrm{HNOCM}=$ hypertrophe nichtobstruktive Kardiomyopathie, EF= linksventrikuläre Ejektionsfraktion, LVEED= linksventrikulärer enddiastolischer Durchmesser, $\mathrm{SR}=$ Sinusrhythmus, VC= Vitalkapazität, FEV1= forciertes exspiratorisches Volumen in einer Sekunde. 
Tabelle H: Polysomnografische Daten

\begin{tabular}{|c|c|c|c|c|c|c|}
\hline Pat. & TST (min) & REM (min) & $\mathrm{AHI}(\# / \mathrm{h})$ & Zentraler Al (\#/h) & Mitt. SaO2 (\%) & Arousalindex \\
\hline$\overline{1}$ & 234,5 & 32,0 & 3,6 & 0,4 & 94 & 20,5 \\
\hline 2 & 461,0 & 78,0 & 51,6 & 45,9 & 90 & 32,9 \\
\hline 3 & 325,0 & 29,5 & 17,0 & 0,7 & 92 & 19,4 \\
\hline 4 & 318,0 & 12,5 & 21,6 & 3,6 & 91 & 37,4 \\
\hline 5 & 326,5 & 30,0 & 15,8 & 6,6 & 91 & 25,7 \\
\hline 6 & 372,0 & 91,0 & 36,5 & 12,0 & 93 & 25,2 \\
\hline 7 & 334,0 & 36,5 & 9,1 & 0,0 & 86 & 26,8 \\
\hline 8 & 399,5 & 76,5 & 8,6 & 5,1 & 91 & 17,1 \\
\hline 9 & 349,5 & 32,0 & 54,9 & 34,1 & 86 & 28,0 \\
\hline 10 & 131,5 & 19,0 & 7,3 & 4,4 & 86 & 84,4 \\
\hline 11 & 337,5 & 60,0 & 25,9 & 13,1 & 92 & 16,5 \\
\hline 12 & 331,0 & 102,0 & 8,8 & 2,0 & 91 & 22,3 \\
\hline 13 & 330,5 & 12,0 & 18,4 & 11,0 & 87 & 10,7 \\
\hline 14 & 256,5 & 0,0 & 40,5 & 38,9 & 73 & 18,5 \\
\hline 15 & 380,0 & 57,5 & 60,5 & 6,8 & 87 & 24,3 \\
\hline 16 & 278,0 & 73,5 & 5,7 & 1,4 & 91 & 44,7 \\
\hline 17 & 300,0 & 0,0 & 10,2 & 0,0 & 88 & 7,4 \\
\hline 18 & 314,0 & 57,5 & 6,1 & 0,2 & 93 & 26,2 \\
\hline 19 & 337,5 & 51,0 & 25,2 & 17,4 & 91 & 37,5 \\
\hline 20 & 254,5 & 38,0 & 31,1 & 18,0 & 88 & 33,2 \\
\hline 21 & 442,0 & 78,5 & 6,2 & 0,1 & 86 & 31,1 \\
\hline 22 & 383,0 & 39,0 & 52,6 & 33,3 & 85 & 23,8 \\
\hline 23 & 138,0 & 22,5 & 1,8 & 0,0 & 93 & 9,6 \\
\hline 24 & 360,5 & 68,5 & 7,6 & 0,0 & 87 & 79,5 \\
\hline 25 & 155,5 & 0,0 & 4,4 & 0,0 & 89 & 32,4 \\
\hline 26 & 248,0 & 55,0 & 13,8 & 0,0 & 88 & 8,7 \\
\hline 27 & 331,5 & 71,5 & 21,7 & 12,8 & 89 & 6,2 \\
\hline 28 & 385,5 & 80,0 & 1,0 & 0,1 & 91 & 28,0 \\
\hline 29 & 323,0 & 30,0 & 20,1 & 11,7 & 94 & 10,8 \\
\hline 30 & 363,0 & 70,5 & 15,8 & 0,5 & 86 & 51,6 \\
\hline 31 & 266,0 & 58,0 & 18,8 & 8,1 & 91 & 43,3 \\
\hline 32 & 192,0 & 18,5 & 2,7 & 0,0 & 93 & 32,2 \\
\hline 33 & 252,0 & 80,0 & 11,4 & 3,9 & 92 & 13,8 \\
\hline 34 & 214,5 & 22,5 & 5,0 & 0,2 & 88 & 32,4 \\
\hline 35 & 237,5 & 40,0 & 26,0 & 8,7 & 92 & 28,3 \\
\hline 36 & 366,5 & 49,0 & 4,2 & 0,2 & 91 & 16,2 \\
\hline 37 & 206,5 & 20,5 & 36,5 & 28,0 & 92 & 20,6 \\
\hline 38 & 376,5 & 83,5 & 3,6 & 0,0 & 88 & 28,4 \\
\hline 39 & 421,5 & 41,0 & 13,3 & 3,5 & 89 & 10,7 \\
\hline 40 & 327,0 & 86,5 & 19,0 & 2,0 & 90 & 19,6 \\
\hline 41 & 332,0 & 105,5 & 42,8 & 41,4 & 87 & 4,9 \\
\hline 42 & 187,0 & 27,5 & 0,8 & 0,0 & 93 & 14,8 \\
\hline 43 & 291,5 & 69,5 & 29,7 & 18,1 & 93 & 27,6 \\
\hline 44 & 167,0 & 39,0 & 11,2 & 0,9 & 95 & 25,7 \\
\hline 45 & 300,5 & 96,0 & 1,6 & 0,0 & 94 & 20,4 \\
\hline 46 & 388,5 & 94,5 & 2,9 & 0,4 & 84 & 40,3 \\
\hline 47 & 314,0 & 69,5 & 8,6 & 2,6 & 91 & 26,8 \\
\hline 48 & 342,5 & 33,0 & 5,0 & 0,0 & 90 & 26,3 \\
\hline 49 & 363,0 & 46,5 & 35,1 & 32,6 & 93 & 22,1 \\
\hline 50 & 329,5 & 57,5 & 4,2 & 2,1 & 91 & 30,4 \\
\hline
\end{tabular}

TST $=$ Total Sleep Time, REM= Rapid Eye Movement, AHI= Apnoe-Hypopnoe-Index, Al= Apnoe-Index, Mitt. SaO2= mittlere Sauerstoffsättigung. 


\subsection{Patientenaufklärung zur Studie}

Georg-August-Universität Göttingen • Bereich Humanmedizin Universitätsklinikum • Medizinische Fakultät

Abteilung Kardiologie und Pneumologie

Direktor: Professor Dr. med. G. Hasenfuß

Leiter Bereich Pneumologie: Prof. Dr. med. Stefan Andreas

Abtl. Kardiologie u. Pneumologie, R. Koch Str. 40, 37075 Göttingen Prof. Dr. S. Andreas

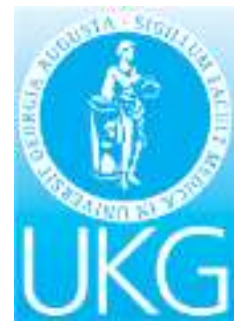

Bronchoskopie, Lungenfunktion Schlaflabor

Tel: $+49-551-39-8872$

Fax:+49-551-39-14370

Zentrale : $-39-0$

Email:Sandreas@med. uni-goettingen.de

Sehr geehrte Patientin,

Sehr geehrter Patient,

Sie leiden an einer schweren Pumpschwäche des Herzens, welche auch als Herzinsuffizienz bezeichnet wird. Bei dieser Erkrankung können besonders nachts Atemstörungen auftreten, die zum Teil mit einer deutlichen Leistungsminderung verbunden sind. Der Einfluss dieser Atemstörung auf die Herzinsuffizienz wird noch nicht gut verstanden. Durch eine Schlaflaboruntersuchung (Polysomnografie) soll das Vorliegen einer Atemstörung im Schlaf untersucht werden. Ziel der Untersuchung ist es, die Häufigkeit des Auftretens der Atemstörung bei Patienten mit Herzinsuffizienz zu bestimmen. Die Untersuchung im Schlaflabor wird folgendermaßen durchgeführt: Nachdem sie mit den örtlichen Gegebenheiten des Schlaflabors vertraut gemacht wurden, wird eine alkoholische Säuberung der für die Elektrodenanbringung vorgesehenen Hautpartien durchgeführt. Im Weiteren werden Elektroden für die Aufzeichnung der Hirnstromkurven, der Augenbewegungen, der Muskelaktivität, der Herzaktionen und der Atmung einschließlich der Sauerstoffsättigung angebracht. Nach dem Zu-Bett-Gehen werden die Elektroden mit dem Computer des Schlaflabors verbunden. Zur weiteren Überprüfung der Ableitungsqualität werden Bewegungsmanöver für die verschiedenen Elektroden durchgeführt. Während der Nacht ist ein Beobachter zur Überwachung in einem Nebenraum anwesend. Die Polysomnografie ist eine wissenschaftliche Untersuchung. Einen Vorteil durch die Teilnahme an der Studie gewinnen Sie durch die Abklärung möglicher Atemstörungen im Schlaf.

Wenn sie wünschen, teilen wir Ihnen gerne Ihre Ergebnisse mit. Ihre persönlichen Daten bleiben geschützt. Die Auflagen des Datenschutzes werden beachtet. Sie können jederzeit ohne Angabe von Gründen und ohne Nachteile für Ihre weitere Behandlung von der Studie zurücktreten. 


\subsection{Einverständniserklärung des Patienten}

Georg-August-Universität Göttingen • Bereich Humanmedizin

Universitätsklinikum • Medizinische Fakultät

Abteilung Kardiologie und Pneumologie

Direktor: Professor Dr. med. G. Hasenfuß

Leiter Bereich Pneumologie: Prof. Dr. med. Stefan Andreas

Abtl. Kardiologie u. Pneumologie, R. Koch Str. 40, 37075 Göttingen

Prof. Dr. S. Andreas

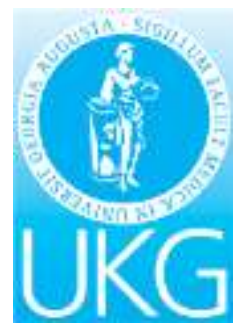

Bronchoskopie,Lungenfunktion Schlaflabor

Tel: +49-551-39-8872

Fax:+49-551-39-14370

Zentrale : -39-0

Email:Sandreas@med.

uni-goettingen.de

Patienteneinverständniserklärung

Ich wurde von meinem Arzt vollständig über Wesen, Bedeutung und Tragweite der klinischen Prüfung aufgeklärt. Ich habe den Aufklärungstext gelesen und verstanden. Ich hatte die Möglichkeit, Fragen zu stellen und habe die Antworten verstanden und akzeptiere sie. Mein Arzt hat mich über die mit der Teilnahme an der Studie verbundenen Risiken und den möglichen Nutzen informiert.

Ich hatte ausreichend Zeit, mich zur Teilnahme an der Studie zu entscheiden und weiß, dass die Teilnahme an der Studie freiwillig ist. Ich weiß, dass ich jederzeit und ohne Angabe von Gründen diese Zustimmung widerrufen kann, ohne dass sich dieser Entschluss nachteilig auf die spätere Behandlung durch meinen Arzt auswirken wird. Mir ist bekannt, dass meine persönlichen Daten in verschlüsselter Form gespeichert werden.

Mit meinem Einverständnis zur Teilnahme erkläre ich gleichzeitig, dass ich mit der im Rahmen dieser klinischen Prüfung erfolgten Aufzeichnung von Krankheitsdaten und ihrer Weitergabe zur Überprüfung an die zuständigen Überwachungsbehörden einverstanden bin.

Ich habe eine Kopie der Patienteninformation und dieser Einwilligungserklärung erhalten. Ich erkläre hiermit die freiwillige Teilnahme an dieser klinischen Studie.

Göttingen, den

Unterschrift 


\subsection{Abkürzungen}

AHI

Al

ASV

BMI

CPAP

CSA

EEG

EF

EKG

EMG

EOG

FEV1

LA

LVEDD

NREM

NYHA

$\mathrm{pCO}_{2}$

$\mathrm{pO}_{2}$

REM

RV

S1-4

TLC

TST

VC

WK
Apnoe-Hypopnoe-Index

Apnoe-Index

Adaptive Servo-Ventilation

Body-Mass-Index

Continuous Positive Airway Pressure

Cheyne-Stokes-Atmung

Elektroenzephalogramm

Linksventrikuläre Ejektionsfraktion

Elektrokardiogramm

Elektromyogramm

Elektrookulogramm

Forcierte exspiratorische Einsekundenkapazität

Durchmesser des linken Vorhofes

Linksventrikulärer enddiastolischer Durchmesser

Non Rapid Eye Movement

New York Heart Association

Kohlendioxidpartialdruck

Sauerstoffpartialdruck

Rapid Eye Movement

Residualvolumen

Schlafstadium 1-4

Totale Lungenkapazität

Total Sleep Time

Vitalkapazität

Wachstadium 


\section{Literaturverzeichnis}

AASM: ICSD-2 International classification of sleep disorders 2nd edn:

Diagnostic and coding manual. Westchester, Illinois/USA 2005

Ancoli-Israel S, Engler RL, Friedman PJ, Klauber MR, Ross PA, Kripke DF (1994): Comparison of Patients With Central Sleep Apnea. With and Without Cheyne-Stokes Respiration. Chest 106, 780-786

Andreas S, Clemens C, Sandholzer H, Figulla HR, Kreuzer H (1996 a): Improvement of Exercise Capacity With Treatment of Cheyne-Stokes Respiration in Patients With Congestive Heart Failure. J Am Coll Cardiol $\underline{27}$, 1486-1490

Andreas S, Hagenah G, Möller C, Werner GS, Kreuzer H (1996 b): CheyneStokes Respiration and Prognosis in Congestive Heart Failure. Am J Cardiol $\underline{78}$, 1260-1264

Andreas S, von zur Mühlen F, Stevens J, Kreuzer H (1998 a): Nocturnal oxygen and hypercapnic response in patients with congestive heart failure. Respir Med 92, 426-431

Andreas S, Weidel K, Hagenah G, Heidl S (1998 b): Treatment of CheyneStokes respiration with nasal oxygen and carbon dioxide. Eur Respir J 12, 414419

Andreas S, Reiter H, Lüthje L, Delekat A, Grunewald RW, Hasenfuss G, Somers VK (2004): Differential Effects of Theophylline on Sympathetic Excitation, Hemodynamics, and Breathing in Congestive Heart Failure. Ciculation $\underline{110}, 2157-2162$

Arzt M, Bradley TD (2006): Treatment of sleep apnea in heart failure. Am J Respir Crit Care Med 173, 1300-1308 
Arzt M, Schulz M, Wensel R, Montalvan S, Blumberg FC, Rieger GA, Pfeifer M (2005): Nocturnal continuous positive airway pressure improves ventilatory efficiency during exercise in patients with chronic heart failure. Chest $\underline{127}, 794-$ 802

Arzt M, Floras J, Logan A, Ryan C, Tomlinson G, Bradley T (2006): The CANPAP trial: Effects of CPAP on central sleep apnea, cardiovascular function and mortality in heart failure. Sleep Med $\underline{7}, 19$

Arzt M, Floras JS, Logan AG, Kimoff RJ, Series F, Morrison D, Ferguson K, Belenkie I, Pfeifer M, Fleetham J et al. (CANPAP Investigators) (2007):

Suppression of central sleep apnea by continuous positive airway pressure and transplant-free survival in heart failure: a post hoc analysis of the Canadian Continuous Positive Airway Pressure for Patients with Central Sleep Apnea and Heart Failure Trial (CANPAP). Circulation 115, 3140-3142

ASDA Task Force (1992): EEG Arousels: Scoring Rules and Examples. Sleep $\underline{15}, 173-184$

Berger AJ, Mitchell RA, Severinghaus JW (1977): Regulation of Respiration. New Engl J Med 297, 194-201

Birbaumer N, Schmidt RF: Wachen, Aufmerksamkeit und Schlafen; in: Physiologie des Menschen; hrsg. v. Schmidt RF, Thews G; Springer-Verlag, Berlin/Heidelberg 1997, 141-153

Bradley TD (1992): Right and Left ventricular functional impairment and sleep apnea. Clin Chest Med $\underline{13}$, 459-479.

Bradley TD, Floras JS (2009): Obstructive sleep apnoea and its cardiovascular consequences. Lancet $\underline{373}, 82-93$ 
Bradley TD, Logan AG, Kimoff RJ, Sériès F, Morrison D, Ferguson K, Belenkie I, Pfeifer M, Fleetham J, Hanly P et al. (CANPAP Investigators) (2005): Continuous Positive Airway Pressure for Central Sleep Apnea and Heart Failure. N Engl J Med $\underline{353}$, 2025-2033

Brown HW, Plum F (1961): The Neurologic Basis of Cheyne-Stokes Respiration. Am J Med 1961, 849-860

Buckle P, Millar T, Kryger M (1992): The Effect of Short-term Nasal CPAP on Cheyne-Stokes Respiration in Congestive Heart Failure. Chest 102, 31-35

Campbell SC, Lauver GL, Cobb RB (1981): Central ventilatory depression by oral propranolol. Clin Pharmacol Ther $\underline{30}$ (6), 758-764

CAPRICORN Investigators (2001): Effect of carvedilol on outcome after myocardial infarction in patients with left ventricular dysfunction: the CAPRICORN randomised trial. Lancet $\underline{357}, 1385-1390$

Chapmann KR, Bruce EN, Gothe B, Cherniack NS (1988): Possible mechanisms of periodic breathing during sleep. J Appl Physiol 64, 1000-1008 Cherniak NS, Longobardo GS (1970): Oxygen and Carbon Dioxide Gas Stores of the Body. Physiol Rev $\underline{50}, 196-243$

Cherniak NS, Longobardo GS (1973): Cheyne-Stokes Breathing. An Instability in Physiologic Control. New Engl J Med $\underline{288}$, 952-957

Chernick V, Avery ME (1966): Response of premature infants with periodic breathing to ventilatory stimuli. J Appl Physiol 21, 434-440

Cheyne J (1818): A case of apoplexy, in which the flashy part of the heart was converted into fat. Dublin Hosp Rep 2, 216-223 
Churchill ED, Cope O (1929): The rapid shallow breathing resulting from pulmonary congestion and edema. J Exp Med $\underline{49}, 531-537$

CIBIS Investigators and Committees (1994): A Randomized Trial of $\beta$-Blocker in Heart Failure. The Cardiac Insufficiency Bisoprolol Study (CIBIS). Circulation $\underline{90}, 1765-73$

CONSENSUS Trial Study Group (1987): Effects of enalapril on mortality in severe congestive heart failure: results of the Cooperative North Scandinavian Enalapril Survival Study (CONSENSUS). N Engl J Med $\underline{316}$, 1429-1435

Cowie MR, Wood DA, Coats AJS, Thompson SG, Suresh V, Poole-Wilson PA, Sutton GC (2000): Survival of patients with a new diagnosis of heart failure: a population based study. Heart $\underline{83}, 505-510$

Cross KW, Hooper JMD, Oppe' TE (1953): The effect of inhalation of carbon dioxide in air on the respiration of the full term and premature infant. J Physiol 122, 264-273

Crowell JW, Guyton AC, Moore JW (1956): Basic oscillating mechanism of Cheyne-Stokes respiration. Am J Physiol $\underline{187}$, 395-398

Dark DS, Pingleton SK, Kerby GR, Crabb JE, Gollub SB, Glatter TR, Dunn MI (1987): Breathing Pattern Abnormalities and Arterial Oxygen Desaturation during Sleep in the Congestive Heart Failure Syndrome. Chest $\underline{91}, 833-836$

Davies RJO, Harrington KJ, Ormerod OJM, Stradling JR (1993): Nasal Continous Positive Airway Pressure in Chronic Heart Failure with Sleepdisordered Breathing. Am Rev Respir Dis $\underline{147}$, 630-634

Dempsey JA, Skatrud JB (1986): A Sleep-Induced Apneic Threshold and Its Consequences. Am Rev Respir Dis 133, 1163-1170 
Deutsche Gesellschaft für Kardiologie (2005): Leitlinien zur Therapie der chronischen Herzinsuffizienz. Z Kardiol 94, 488-509.

Digitalis Investigation Group (1997): The effect of digoxin on mortality and morbidity in patients with heart failure. N Engl J Med $\underline{336}, 525-533$

Dowdell WT, Javaheri S, McGinnis W (1990): Cheyne-Stokes Respiration Presenting as Sleep Apnea Syndrome. Am Rev Respir Dis 141, 871-879

Dowell AR, Buckley CE, Cohen R, Whalen RE, Sieker HO, Durham NC (1971): Cheyne-Stokes Respiration. A Review of Clinical Manifestation and Critique of Physiological Mechanisms. Arch Intern Med 127, 712-726

Dyugovskaya L, Lavie P, Lavie L (2002): Increased adhesion molecules expression and production of reactive oxygen species in leukocytes of sleep apnea patients. Am J Respir Crit Care Med 165, 934-939

European Society of Cardiology Task Force (2001): Guidelines for the diagnosis and treatment of chronic heart failure. Eur Heart $\mathrm{J} \underline{22}, 1527-1560$

Ferrier K, Campbell A, Yee B, Richards M, O'Meeghan T, Weatherall M, Neill A (2005): Sleep-disordered breathing occurs frequently in stable outpatients with congestive heart failure. Chest $\underline{128}, 2116-2122$

Flather M, Yusuf S, Kober L (2000): Long-term ACE-Inhibitor Therapy in Patient with Heart Failure or Left Ventricular Dysfunction: a systemic overview of data from individual patients. Lancet $\underline{355}, 1575-1581$

Fox KF, Cowie FR, Wood DA, Coats AJS, Gibbs JSR, Underwood SR, Turner RM, Poole-Wilson PA, Davies SW and Sutton GC (2001): Coronary artery disease as the cause of incident heart failure in the population. Eur Heart $\mathrm{J} \underline{22}$, 228-236 
Franklin KA, Eriksson P, Sahlin C, Lundgren R (1997): Reversal of central sleep apnea with oxygen. Chest $\underline{111}, 163-169$

Garlind T, Linderholm H (1958): Hypoventilation Syndrome in a Case of Chronic Epidemic Encephalitis. Acta Med Scand 162, 333-349

Garrigue S, Bordier P, Jaïs P, Shah DC, Hocini M, Raherison C, Tunon De Lara M, Haïssaguerre M, Clementy J (2002): Benefit of atrial pacing in sleep apnea syndrome. N Engl J Med $\underline{346}$, 404-12.

Gehlbach BK, Geppert E (2004): The pulmonary manifestation of left heart failure. Chest $\underline{125}, 669-682$

Granger CB, McMurray JJ, Yusuf S, Held P, Michelson EL, Olofsson B, Ostergren J, Pfeffer MA, Swedberg K (2003): Effects of candesartan in patients with chronic heart failure and reduced left-ventricular systolic function intolerant to angiotensin-converting-enzyme inhibitors: the CHARM Alternative Trial. Lancet $\underline{362}, 772-776$

Gustafsson F, Torp-Pedersen C, Brendorp B, Seibækb M, Burchardt H, Køberc $L$ (2003): Long-term survival in patients hospitalized with congestive heart failure: relation to preserved and reduced left ventricular systolic function. Eur Heart J $\underline{24}, 863-870$.

Hanly P, Zuberi-Khokar N (1995): Daytime Sleepiness in Patients With Congestive Hart Failure an Cheyne-Stokes Respiration. Chest 107, 952-958

Hanly P, Zuberi-Khokhar S (1996): Increased Mortality Associated with CheyneStokes Respiration in Patients with Congestive Heart Failure. Am J Respir Crit Care Med 153, 272-276

Hanly P, Millar TW, Steljes DG, Baert R, Frais MA, Kryger MH (1989): The Effect of Oxygen on Respiration and Sleep in Patients with Congestive Heart Failure. Ann Intern Med 111, 777-782 
Hanly P, Zuberi N, Gray R (1993): Pathogenesis of Cheyne-Stokes Respiration in Patients With Congestive Heart Failure. Chest 104, 1079-1084

Harrison TR, King CE, Calhoun JA, Harrison WG Jr. (1934): Congestive heart failure: Cheyne Stokes respiration as the cause of paroxysmal dyspnea at the onset of sleep. Arch Intern Med $\underline{53}, 891-910$

Hjalmarson A, Goldstein S, Fagerberg B, Wedel H, Waagstein F, Kjekshus J, Wikstrand J, El Allaf D, Vitovec J, Aldershvile J et al. (2000): Effects of controlled-release metoprolol on total mortality, hospitalizations, and well-being in patients with heart failure: the Metoprolol CR/XL Radomized Intervention Trial in congestive heart failure (MERIT-HF). MERIT-HF Study Group. JAMA 283, 1295-1302

Horner RL, Brooks D, Kozar LF, Tse S, Phillipson EA (1994): Immediate effects of arousel from sleep on cardiac autonomic outflow in the absence of breathing in dogs. J Appl Physiol $\underline{79}$, 151-162

Howard PJ, Bauer AR (1949): Irregularities of Breathing in the Newborn Period. Am J Dis Child $\underline{77}, 592-609$

Javaheri S, Corbett WS (1998): Association of Low PaCO2 with Central Sleep Apnea and Ventricular Arrhythmias in Ambulatory Patients with Stable Heart Failure. Ann Intern Med 128, 204-207

Javaheri S, Parker TJ, Wexler L, Michaels SE, Stanberry E, Nishyama H, Roselle GA (1995): Occult Sleep-Disordered Breathing in Stable Congestive Heart Failure. Ann Intern Med 122, 487-492

Javaheri S, Parker TJ, Wexler L, Liming JD, Lindower P, Roselle GA (1996):

Effect of Theophylline on Sleep-disordered Breathing in Heart Failure.

N Engl J Med $\underline{335}, 562-567$ 
Javaheri S, Parker TJ, Liming JD, Corbett WS, Nishyama H, Wexler L, Roselle GA (1998): Sleep Apnea in 81 Ambulatory Male Patients With Stable Heart Failure. Circulation 97, 2154-2159

Javaheri S, Ahmed M, Perker TJ, Brown CR (1999): Effects of nasal O2 sleepdisordered breathing in ambulatory patients with stable heart failure. Sleep $\underline{22}$ (8), 1101-1106

Karp HR, Sieker HO, Heyman A (1961): Cerebral Circulation and Function in Cheyne-Stokes Respiration. Am J Med 30, 861-870

Khoo MCK, Gottschalk A, Pack Al (1991): Sleep-induced periodic breathing and apnea: a theoretical study. J Appl Physiol $\underline{70}$, 2014-24

Klein O (1930): Untersuchungen über das Cheyne-Stokesche Atmungsphänomen. Verh Dtsch Ges Inn Med 42, 217-222

Köhnlein T, Welte T, Tan LB, Elliott MW (2002): Assisted ventilation for heart failure patients with Cheyne-Stokes respiration. Eur Respir J 므, 934-941

Köhnlein T, Welte T (2007): Does beta-blocker treatment influence central sleep apnoea? Respir Med 101 (4), 850-853

Krachmann SL, D’Alonzo GE, Berger TJ, Eisen HJ (1999): Comparison of oxygen therapy with nasal continuous positive airway pressure on CheyneStokes respiration during sleep in congestive heart failure. Chest $\underline{116}$ (6), 15501557

Lafranchi P, Braghiroli A, Bosimi E, Mazzuero G, Colombo R, Donner CF, Giannuzzi P (1999): Prognostic Value of Nocturnal Cheyne-Stokes Respiration in Chronic Heart Failure. Circulation 99, 1435-1440 
Lafranchi PA, Somers VK, Braghiroli A, Corra U, Eleuteri E, Giannuzzi P (2003): Central sleep apnea in left ventricular dysfunction: prevalence and implications for arrhythmic risk. Circulation $\underline{107}, 727-732$

Lange RL, Hecht HH (1961): The Mechanism of Cheyne-Stokes Respiration. J Clin Invest $\underline{41}, 42-52$

Levy D, Kenchaiah S, Larson MG, Benjamin EJ, Kupka MJ, Ho KKL, Murabito JM, Vasan RS (2002): Long-term trends in the incidence of and survival with heart failure. N Engl J Med $\underline{347}, 1397-1402$.

Likoff MJ, Chandler SL, Kay HR (1987): Clinical Determinants of Mortalitiy in Chronic Congestive Heart Failure Secondary to Idiopathic Dilated or to Ischemic Cardiomyopathy. Am J Cardiol $\underline{59}$, 634-638

Lorenzi-Filho G, Rankin F, Bies I, Bradley D (1999): Effects of Inhaled Carbon Dioxide and Oxygen on Cheyne-Stokes Respiration in Patients with Heart Failure. Am J Respir Crit Care Med 159, 1490-1498

Lüthje L, Renner B, Kessels R, Vollmann D, Raupach T, Gerritse B, Tasci S, Schwab JO, Zabel M, Zenker D et al. (2009): Cardiac resynchronisation therapy and atrial overdrive pacing for the treatment of central sleep apnea. Eur J Heart Fail $\underline{11}, 273-280$

Mak S, Azevedo ER, Liu PP, Newton GE (2001): Effect of hyperoxia on left ventricular function and filling pressures in patients with and without congestive heart failure. Chest $\underline{120}$ (2), 467-473.

Mared L, Cline C, Erhardt L, Berg S, Midgren B (2004): Cheyne-Stokes respiration in patients hospitalised for heart failure. Respir Res $\underline{5}, 14$

McKee P, Castelli WP, McNamara PM, Kannel WB (1971): The Natural History of Congestive Heart Failure: the Framingham Study. N Engl J Med 285, 14411446 
McMurray JJ, Stewart S (2000): Epidemiology, aetiology and prognosis of heart failure. Heart $\underline{83}, 596-602$

Morgenthaler TI, Gay PC, Gordon N, Brown LK (2007): Adaptive servoventilation versus noninvasive positive pressure ventilation for central, mixed, and complex sleep apnea syndromes. Sleep $\underline{30}$, 468-475

Mortara A, Sleight P, Pinna GD, Maestri R, Capomolla S, Febo O, La Rovere MT, Cobelli F (1999): Association between hemodynamic impairment and Cheyne-Stokes respiration and periodic breathing in chronic stable congestive heart failure secondary to ischemic or idiopathic dilated cardiomyopathy. Am J Cardiol $\underline{84}$ (8), 900-4

Naughton MT, Benard D, Tam A, Rutherford R, Bradley TD (1993): Role of Hyperventilation in the Pathogenesis of Central Sleep Apneas in Patients with Congestive Heart Failure. Am Rev Respir Dis $\underline{148}$, 330-338

Naughton MT, Liu PP, Benard DC, Goldstein RS, Bradley TD (1995): Treatment of Congestive Heart Failure and Cheyne-Stokes Respiration by Continuous Positive Airway Pressure. Am J Respir Crit Care Med 151, 92-97

NYHA: Diseases of the heart and the blood vessels. Nomenclature and criteria for diagnosis by THE Criteria Committee of the New York Heart Association. 6 . Auflage Little, Brown and Company, Boston 1964

Oldenburg O, Faber L, Vogt J, Dorszewski A, Szabados F, Horstkotte D, Lamp B (2007): Influence of cardiac resynchronisation therapy on different types of sleep disordered breathing. Eur J Heart Fail $\underline{9}$, 820-826

Oltmanns KM, Gehring H, Rudolf S, Schultes B, Rook S, Schweiger U, Born J, Fehm HL, Peters A (2004): Hypoxia Causes Glucose Intolerance in Humans. Am J Resp Crit Care Med 169, 1231-1237 
Pepperell JC, Maskell NA, Jones DR, Langford-Wiley BA, Crosthwaite N, Stradling JR, Davies RJ (2003): A randomised controlled trial of adaptive ventilation for Cheyne-Stokes breathing in heart failure. Am J Respir Crit Care Med $\underline{168}$ (9), 1109-1114

Pevernagie D, Janssens JP, De Backer W, Elliot M, Pepperell J, Andreas S (2007): Ventilatory support and pharmacological treatment of patients with central apnoea or hypoventilation during sleep. Eur Respir Rev 16, 115-124

Pfeffer MA, Braunwald E, Moyé LA, Basta L, Brown EJ, Cuddy TE, Davis BR, Geltman EM, Goldmann S, Flaker GC et al. (1992): Effect of Captopril on Mortality and Morbidity in Patients with Left Ventricular Dysfunction after Myocardial Infarction. N Engl J Med $\underline{327}$, 669-677

Philippe C, Stoica-Herman M, Drouot X, Raffestin B, Escourrou P, Hittinger L, Michel PL, Rouault S, d'Ortho MP (2006): Compliance with and effectiveness of adative servoventilation versus continuous positive airway pressure in the treatment of Cheyne-Stokes respiration in heart failure over a six month period. Heart $\underline{92}$ (3), 337-342

Pitt B, Zannad F, Remme WJ, Cody R, Castaigne A, Perez A, Palensky J, Wittes $J$ (1999): The effect of spironolactone on morbidity and mortality in patients with severe heart failure. Randomized Aldactone Evaluation Study (RALES) Investigators. N Engl J Med $\underline{341}, 709-717$

Pitt B, Poole-Wilson PA, Segal R, Martinez FA, Dickstein A, Camm AJ, Riegger G, Klinger GH, Neaton J, Sharma D et al. (2000): Effect of losartan compared with captopril on mortality in patients with symptomatic heart failure: randomised trial- the Losartan Heart Failure Survival Study ELITE II. Lancet $\underline{355}$, 1582-1587

Pitt B, Remme W, Zannad F, Neaton J, Martinez F, Roniker B, Bittman R, Hurley S, Kleiman J, Gatlin M (2003): Eplerenone, a selective aldosterone blocker, in patients with left ventricular dysfunction after myocardial infarction. $\mathrm{N}$ Engl J Med $\underline{348}, 1309-1321$ 
Pryor WW (1951): Cheyne-Stokes Respiration in Patients with Cardiac Enlargement and Prolonged Circulation Time. Circulation 4, 233-238

Rathore SS, Curtis JP, Wang Y, Bristow MR, Krumholz HM (2003): Association of serum digoxin concentration and outcomes in patients with heart failure. JAMA $\underline{289}, 871$ - 878

Rechtschaffen A, Kales A: A Manual of Standarized Terminology, Techniques and Scoring System for Sleep Stages of Human Subjects. (National Institutes of Health Publication, No. 204). National Institute of Health, Bethesda 1968

Rihal CS, Davis KB, Kennedy JB, Gersh BJ (1995): The utility of clinical, electrocardiographic, and roentgenographic variables in the prediction of left ventricular function. Am J Cardiol $\underline{75}$, 220-223

Roebuck T, Solin P, Kaye DM, Bergin P, Bailey M, Naughton T (2004): Increased long-term mortality in heart failure due to sleep apnoea is not yet proven. Eur Respir J $\underline{23}$ (5), 735-740

Schädlich S, Königs I, Kalbitz F, Blankenburg T, Busse HJ, Schütte W (2004): Kardiale Leistungsfähigkeit bei Patienten mit Cheyne-Stokes-Atmung infolge Herzinsuffizienz während langfristiger nasaler Beatmungstherapie mittels adaptiver Servoventilation. Z Kardiol $\underline{93}$, 454-462

Schulz R, Blau A, Börgel J, Duchna HW, Fietze I, Koper I, Prenzel R, Schädlich $S$, Schmitt J, Tasci S, Andreas S for the working group "Kreislauf und Schlaf" of the German Sleep Society (DGSM) (2007): Sleep apnoea in heart failure. Eur Respir J $\underline{29}$ 2007, 1201-1205

Sin DD, Fitzgerald F, Parker JD, Newton G, Floras JS, Bradley TD (1999): Risk Factors for Central and Obstructive Apnea in 450 Men And Women with Congestive Heart Failure. Am J Respir Crit Care Med 160, 1101-1106 
Sin DD, Logan AG, Fitzgerald FS, Liu PP, Bradley TD (2000): Effects of Continuous Positive Airway Pressure on Cardiovascular Outcomes in Heart Failure Patients With and Without Cheyne-Stokes Respiration. Circulation 102, 61-66

Sinha AM, Skobel EC, Breithardt OA, Norra C, Markus KU, Breuer C, Hanrath $P$, Stellbrink $C$ (2004): Cardiac resynchronization therapy improves central sleep apnea and Cheyne-Stokes respiration in patients with chronic heart failure. $J$ Am Coll Cardiol $\underline{44}, 68-71$

SOLVD Investigators (1999): Effect of Enalapril on Mortality and Development of Heart Failure in Asymptomatic Patients with reduced Left Ventricular Ejection Fraction. N Engl J Med 327, 685-691

Somers VK, Dyken ME, Mark AL, Abboud F (1993): Sympathetic-Nerve Activity during Sleep in normal subjects. N Engl J Med $\underline{328}, 303-307$

Somers VK, Dyken ME, Clary MP, Abboud FM (1995): Sympathetic neural mechanisms in obstructive sleep apnea. J Clin Invest $\underline{96}, 1897-1904$

Spiegel K, Leproult R, Van Cauter E (1999): Impact of sleep debt on metabolic and endocrine function. Lancet $\underline{354}$, 1435-1439

Staniforth AD, Kinnear WJ, Starlin R, Hetmanski DJ, Cowley AJ (1998): Effect of oxygen on sleep quality, cognitive function and sympathetic activity in patients with chronic heart failure and Cheyne-Stokes respiration. Eur Heart J $\underline{19}, 922-$ 928

Stokes W : The Diseases Of The Heart And The Aorta. Hodges and Smith, Dublin 1854.

Suissa S, Hemmelgarn B, Blais L, Ernst P (1996): Bronchodilators and acute cardiac death. Am J Respir Crit Care Med 154, 1598-1602 
Tamura A, Kawano Y, Naono S, Kotoku M, Kadota J (2007): Relationship between beta-blocker treatment and the severity of central sleep apnea in chronic heart failure. Chest 131 (1), 130-135

Teschler H, Döhring J, Wang YM, Berthon-Jones M (2001): Adaptive Pressure Support Servo-Ventilation. A Novel Treatment for Cheyne- Stokes Respiration in Heart Failure. Am J Respir Crit Care Med 164, 614-619

Töpfer V, El Sebai M, Wessendorf TE, Moraidis I, Teschler H (2004): Adaptive servoventlation: effect on Cheyne-Stokes respiration and on quality of life. Pneumologie $\underline{58}, 28-32$

TRACE Study Group (1995): Effects on Mortality by Tandolapril after Myocardial Infarction. N Engl J Med $\underline{333}, 1670-1676$

Tremel F, Pépin JL, Veale D, Wuyam B, Siché JP, Mallion JM, Lévy P (1999): High prevalence and persistence of sleep apnoea in patients referred for acute left ventricular failure and medically treated over 2 months. Eur Heart $\mathrm{J} \underline{20}$, $1201-1209$

Vongpatanasin W, Thomas GD, Schwartz R, Cassis LA, Osborne-Lawrence S, Hahner L, Gibson LL, Black S, Samols D, Shaul PW (2007): C-Reactive Protein Causes Downregulation of Vascular Angiotensin Subtype 2 Receptors and Systolic Hypertension in Mice. Circulation 115, 1020-1028

Walsh JT, Andrews R, Starling R, Cowley AJ, Johnston ID, Kinnear WJ (1995): Effects of Captopril and oxygen on sleep apnoea in patients with mild to moderate congestive cardiac failure. $\mathrm{Br}$ Heart $\mathrm{J} \underline{73}, 237-241$

Younes M (1989): The Physiologic Basis of the Central Apnea and Periodic Breathing. Curr Pulmonol 10, 265-326 


\section{Danksagungen}

Ich danke Herrn Prof. Dr. med. Stefan Andreas für die Überlassung des Themas meiner Promotion und die Hilfe bei deren Fertigstellung.

Herrn Dr. med. Gerrit Hagenah danke ich für die Hilfe bei der Durchführung der Studie und die hilfreichen Korrekturen meiner Arbeit, häufig auch unter widrigen Bedingungen. 


\section{Lebenslauf}

Geboren wurde ich am 6. Januar 1979 in Berlin-Charlottenburg.

Meine Schulzeit schloss ich 1998 mit dem Abitur am Käthe-Kollwitz-Gymnasium in Wilhelmshaven ab.

Dem Zivildienst beim Malteser Hilfsdienst in Wilhelmshaven folgte vom Oktober 1999 bis zum März 2006 das Studium der Medizin an der Georg-AugustUniversität zu Göttingen.

Vom Juni 2006 bis zum Mai 2010 war ich unter der Leitung von Herrn Dr. med. Aloys Klaus, Herrn Prof. Dr. med. Jochen Schrader und Herrn PD Dr. med. Jörg Bäsecke als Assistenzarzt in der Abteilung für Innere Medizin des St. JosefsHospitals in Cloppenburg tätig. Seit dem Juli 2010 setze ich meine internistische Ausbildung in der Abteilung von Frau Dr. med. Regina Prenzel im Pius Hospital Oldenburg fort.

Im Mai 2010 heiratete ich meine Frau Valerie Escher. Zusammen leben wir in Oldenburg. 